Supporting Information

\title{
Highly Efficient Recognition of Native TpT by Artificial Ditopic Hydrogen-Bonding Receptors Possessing a Conformationally Well- Defined Linkage
}

\author{
Masayoshi Takase ${ }^{\dagger}$ and Masahiko Inouye* \\ Faculty of Pharmaceutical Sciences, Toyama Medical and Pharmaceutical University, \\ Toyama 930-0194, Japan \\ ${ }^{\dagger}$ Department of Chemistry, Graduate School of Science, Kyoto University, \\ Kyoto 606-8502, Japan
}

Synthetic procedures and spectroscopic data (mp, IR, ${ }^{1} \mathrm{H}$ NMR, ${ }^{13} \mathrm{C}$ NMR, MS, and elemental analysis)

Lipophilic TpT Analogue 3. To a THF (13 mL) solution of dichlorodi- $n$ hexylsilane $(404 \mathrm{mg}, 1.50 \mathrm{mmol})$ and pyridine $(3.1 \mathrm{~mL})$ was added a THF $(10 \mathrm{~mL})$ solution of 5'-O-(tert-butyldimethylsilyl)thymidine ${ }^{23}(500 \mathrm{mg}, 1.40 \mathrm{mmol})$ dropwise at $78{ }^{\circ} \mathrm{C}$ over a 30 min period. After the reaction mixture was stirred at $-78{ }^{\circ} \mathrm{C}$ for $2 \mathrm{~h}$, to the mixture was added a THF $(10 \mathrm{~mL})$ solution of 3'-O-(tertbutyldimethylsilyl)thymidine ${ }^{23}(463 \mathrm{mg}, 1.30 \mathrm{mmol})$ dropwise at the same temperature over a $10 \mathrm{~min}$ period. The reaction mixture was then allowed to warm slowly to room temperature with stirring and evaporated. The residue was poured into brine and extracted with $\mathrm{CHCl}_{3}$. The $\mathrm{CHCl}_{3}$ extract was evaporated and chromatographed (silica gel; eluent, $\mathrm{CH}_{2} \mathrm{Cl}_{2} / \mathrm{MeOH} 30: 1$ ) to afford crude 3 . The crude 3 was purified by HPLC (ODS; eluent, $\mathrm{MeOH})$ to give pure 3: yield 15\% (193 mg); mp 48-50 ${ }^{\circ} \mathrm{C}$; IR (KBr) 3419, 
1699, 1277, $1255 \mathrm{~cm}^{-1} ;{ }^{1} \mathrm{H} \mathrm{NMR}\left(\mathrm{CDCl}_{3}\right) \delta 8.87$ (br s, $\left.2 \mathrm{H}\right), 7.47$ (br s, $\left.1 \mathrm{H}\right), 7.41$ (br s, 1 H), 6.38-6.24 (m, $2 \mathrm{H}), 4.55-4.46(\mathrm{~m}, 1 \mathrm{H}), 4.42-4.33(\mathrm{~m}, 1 \mathrm{H}), 4.00$ (br s, $1 \mathrm{H})$, 3.96-3.69 (m, 5 H), 2.34-2.22 (m, 2 H), 2.09-1.97 (m, 2 H), 1.92 (s, 6 H), 1.40-1.21 (m, $16 \mathrm{H}), 0.97-0.82$ (m, $24 \mathrm{H}), 0.70-0.62$ (m, $4 \mathrm{H}), 0.114$ (s, 3H), 0.109 (s, 3H), 0.82 (s, 3H), $0.77(\mathrm{~s}, 3 \mathrm{H}) ;{ }^{13} \mathrm{C} \mathrm{NMR}\left(\mathrm{CDCl}_{3}\right) \delta 163.66,150.22,135.52,135.21,110.92,110.77,87.77$, $87.12,84.93,84.86,72.88,71.85,63.33,62.20,41.20,40.89,33.04,33.01,31.39$, 25.87, $25.68,22.71,22.66,22.52,18.33,17.93,14.05,12.64,12.51,12.46,-4.68,-4.84,-5.37$, 5.54; FABMS (in 3-nitrobenzyl alcohol) $m / e$ (rel intensity) $909\left(\mathrm{MH}^{+}, 100 \%\right)$. Anal. Calcd for $\mathrm{C}_{46} \mathrm{H}_{82} \mathrm{~N}_{2} \mathrm{O}_{10} \mathrm{Si}_{3}$ : C, 60.89; H, 9.11; N, 3.09 Found: C, 59.34; H, 8.87; N, 5.80.

Naphthalene-Linked U-U Dinucleotide Derivative 5. To a $\mathrm{DMF}_{-} \mathrm{Et}_{2} \mathrm{NH}(2+$ $2 \mathrm{~mL}$ ) mixed solution of $\mathbf{2 6}^{19}$ (152 mg, $\left.0.40 \mathrm{mmol}\right),\left(\mathrm{Ph}_{3} \mathrm{P}\right)_{2} \mathrm{PdCl}_{2}(28 \mathrm{mg}, 40 \mu \mathrm{mol})$, and CuI (3.8 mg, $20 \mu \mathrm{mol})$ was added a DMF (2 mL) solution of 25 (248 mg, $1.0 \mathrm{mmol})$ dropwise at $0{ }^{\circ} \mathrm{C}$. The reaction mixture was stirred at room temperature for $5 \mathrm{~h}$ and evaporated. The residue was poured into water and extracted with $\mathrm{CH}_{2} \mathrm{Cl}_{2}$. The $\mathrm{CH}_{2} \mathrm{Cl}_{2}$ extract was evaporated and chromatographed (silica gel; eluent, ether/hexane 4:1) to give 5: yield 24\% (150 mg); mp 174-175 ${ }^{\circ} \mathrm{C}$; IR (KBr) 3419, $1699 \mathrm{~cm}^{-1} ;{ }^{1} \mathrm{H} \mathrm{NMR}\left(\mathrm{CDCl}_{3}\right) \delta$ 9.84 (s, 2 H), 7.83 (d, J = 7.8 Hz, 2 H), 7.80 (d, J = 7.1 Hz, 2 H), 7.57 (s, 2 H), 7.44 (dd, J $=7.8,7.1 \mathrm{~Hz}, 2 \mathrm{H}), 3.78(\mathrm{t}, J=7.3 \mathrm{~Hz}, 4 \mathrm{H}), 1.77-1.64(\mathrm{~m}, 4 \mathrm{H}), 1.40-1.15(\mathrm{~m}, 20 \mathrm{H})$, $0.86(\mathrm{t}, J=6.5 \mathrm{~Hz}, 6 \mathrm{H}) ;{ }^{13} \mathrm{C} \mathrm{NMR}\left(\mathrm{CDCl}_{3}\right) \delta 161.33,150.61,147.40,134.93,133.90$, $132.25,130.08,125.51,120.09$, 100.89, 92.90, 87.33, 49.17, 31.74, 29.14, 29.04, 26.49, 22.60, 14.07; FABMS (in 3-nitrobenzyl alcohol) $m / e$ (rel intensity) $621\left(\mathrm{MH}^{+}, 100 \%\right)$. Anal. Calcd for $\mathrm{C}_{38} \mathrm{H}_{44} \mathrm{~N}_{4} \mathrm{O}_{4}$ : C, 73.52; H, 7.14; N, 9.03. Found: C, 73.58; H, 7.02; N, 8.81.

Biphenylene-Linked U-U Dinucleotide Derivative 6. $\mathrm{An}_{3} \mathrm{~N}(11 \mathrm{~mL})$ solution of 25 (738 mg, $2.97 \mathrm{mmol}), 28$ (300 mg, $0.74 \mathrm{mmol}),\left(\mathrm{Ph}_{3} \mathrm{P}\right)_{2} \mathrm{PdCl}_{2}(13 \mathrm{mg}, 18$ $\mu \mathrm{mol})$, and $\mathrm{CuI}(3.4 \mathrm{mg}, 18 \mu \mathrm{mol})$ was stirred at $40{ }^{\circ} \mathrm{C}$ for $1 \mathrm{~h}$. After removal of the solvent, the residue was recrystallized from $\mathrm{CHCl}_{3}$ to give 6: yield $81 \%(385 \mathrm{mg}) ; \mathrm{mp}$ 237-239 ${ }^{\circ} \mathrm{C}$; IR (KBr) 3446, 2219, 1705, $1689 \mathrm{~cm}^{-1}$; ${ }^{1} \mathrm{H}$ NMR (DMSO-d $) \delta 11.55$ (s, 2 
H), 7.99 (s, 2 H), 6.90-6.76 (m, $6 \mathrm{H}), 3.55(\mathrm{t}, J=7.1 \mathrm{~Hz}, 4 \mathrm{H}), 1.57-1.46(\mathrm{~m}, 4 \mathrm{H})$, $1.30-1.16(\mathrm{~m}, 20 \mathrm{H}), 0.84(\mathrm{t}, J=6.6 \mathrm{~Hz}, 6 \mathrm{H}) ;{ }^{13} \mathrm{C}$ NMR (DMSO-d $\left.{ }_{6}\right) \delta 161.58,150.87$, 149.83, 149.60, 148.95, 131.50, 129.78, 117.80, 112.96, 97.10, 87.84, 85.92, 48.14, 31.20, 28.56, 28.50, 28.23, 25.77, 22.07, 13.93; FABMS (in 3-nitrobenzyl alcohol) m/e (rel intensity) $645\left(\mathrm{MH}^{+}, 100 \%\right)$. Anal. Calcd for $\mathrm{C}_{40} \mathrm{H}_{44} \mathrm{~N}_{4} \mathrm{O}_{4}: \mathrm{C}, 74.51 ; \mathrm{H}, 6.88 ; \mathrm{N}, 8.69$. Found: C, 74.27; H, 6.92; N, 8.80.

Anthracene-Linked U-U Dinucleotide Derivative 7. $\mathrm{A} \mathrm{CH}_{3} \mathrm{CN}-\mathrm{Et}_{3} \mathrm{~N}(7+7$ $\mathrm{mL}$ ) mixed solution of 23 (1.86 g, $5.31 \mathrm{mmol}), \mathbf{2 9}^{21}$ (400 mg, $\left.1.77 \mathrm{mmol}\right),\left(\mathrm{Ph}_{3} \mathrm{P}\right)_{2} \mathrm{PdCl}_{2}$ (31 mg, $44 \mu \mathrm{mol})$, and $\mathrm{CuI}(8 \mathrm{mg}, 44 \mu \mathrm{mol})$ was stirred at $60^{\circ} \mathrm{C}$ for $2 \mathrm{~h}$. After removal of the solvent, the residue was poured into water and extracted with $\mathrm{CHCl}_{3}$. The $\mathrm{CHCl}_{3}$ extract was evaporated and chromatographed (silica gel; eluent, $\mathrm{CH}_{2} \mathrm{Cl}_{2} / \mathrm{MeOH} 40: 1$ ) to give 7: yield 26\% (311 mg); mp 254-255 ${ }^{\circ} \mathrm{C}$; IR (KBr) 3415, 2207, $1687 \mathrm{~cm}^{-1}$; ${ }^{1} \mathrm{H}$ NMR $\left(\mathrm{DMSO}_{-} d_{6}\right) \delta 11.68(\mathrm{~s}, 2 \mathrm{H}), 9.31(\mathrm{~s}, 1 \mathrm{H}), 8.74(\mathrm{~s}, 1 \mathrm{H}), 8.27(\mathrm{~s}, 2 \mathrm{H}), 8.17(\mathrm{~d}, J=8.3 \mathrm{~Hz}$, $2 \mathrm{H}), 7.80(\mathrm{~d}, J=7.0 \mathrm{~Hz}, 2 \mathrm{H}), 7.58(\mathrm{dd}, J=8.3,7.0 \mathrm{~Hz}, 2 \mathrm{H}), 3.70(\mathrm{t}, J=7.1 \mathrm{~Hz}, 4 \mathrm{H})$, 1.67-1.50 (m, $4 \mathrm{H}), 1.35-1.15(\mathrm{~m}, 20 \mathrm{H}), 0.82(\mathrm{~d}, J=6.7 \mathrm{~Hz}, 6 \mathrm{H}) ;{ }^{13} \mathrm{C}$ NMR (DMSO- $\left.d_{6}\right)$ $\delta 161.99,149.92,149.08,131.12,130.99,130.32,129.09,128.17,125.65,122.76,120.53$, $97.49,90.03,88.21,79.17,48.23,31.21,28.58,28.41,25.77,22.07,13.91$; FABMS (in 3nitrobenzyl alcohol) $m / e$ (rel intensity) $671\left(\mathrm{MH}^{+}, 100 \%\right.$ ). Anal. Calcd for $\mathrm{C}_{42} \mathrm{H}_{46} \mathrm{~N}_{4} \mathrm{O}_{4}: \mathrm{C}$, 75.20; H, 6.91; N, 8.35. Found: C, 74.21; H, 6.90; N, 8.34.

Naphthalene-Linked Ditopic Receptor 8. A DMF-Et ${ }_{3} \mathrm{~N}(3+1 \mathrm{~mL})$ mixed solution of 14 (335 mg, $1.11 \mathrm{mmol}), \mathbf{2 6}^{19}$ (141 mg, $\left.0.37 \mathrm{mmol}\right),\left(\mathrm{Ph}_{3} \mathrm{P}\right)_{2} \mathrm{PdCl}_{2}(16 \mathrm{mg}, 22$ $\mu \mathrm{mol})$, and $\mathrm{CuI}(2 \mathrm{mg}, 11 \mu \mathrm{mol})$ was stirred at $55^{\circ} \mathrm{C}$ for $9 \mathrm{~h}$. After removal of the solvent, the residue was poured into water and extracted with $\mathrm{CH}_{2} \mathrm{Cl}_{2}$. The $\mathrm{CH}_{2} \mathrm{Cl}_{2}$ extract was evaporated and chromatographed (silica gel; eluent, $\mathrm{CH}_{2} \mathrm{Cl}_{2} / \mathrm{MeOH} 100: 1$ ) to give 8: yield 74\% (200 mg); mp 170-171 ${ }^{\circ} \mathrm{C}$; IR (KBr) 3295, 2203, 1678, $1553 \mathrm{~cm}^{-1}$; ${ }^{1} \mathrm{H}$ NMR $\left(\mathrm{CDCl}_{3}\right) \delta 7.90-7.84(\mathrm{~m}, 4 \mathrm{H}), 7.76$ (br s, $\left.4 \mathrm{H}\right), 7.63$ (br s, $\left.4 \mathrm{H}\right), 7.49$ (dd, $J=7.8$, $7.5 \mathrm{~Hz}, 2 \mathrm{H}), 2.38-2.28$ (m, $8 \mathrm{H}), 1.72-1.59$ (m, $8 \mathrm{H}), 1.45-1.30$ (m, 8 H), 0.98-0.88 (m, 
$12 \mathrm{H}) ;{ }^{13} \mathrm{C} \mathrm{NMR}\left(\mathrm{CDCl}_{3}\right) \delta 171.62,149.46,136.27,135.59,133.99,131.37,130.56$, $125.84,119.74,112.10,94.66,94.43,37.37,27.58,22.64,14.12$; FABMS (in 3nitrobenzyl alcohol) $m / e$ (rel intensity) $727\left(\mathrm{MH}^{+}, 100 \%\right)$. Anal. Calcd for $\mathrm{C}_{44} \mathrm{H}_{50} \mathrm{~N}_{6} \mathrm{O}_{4}: \mathrm{C}$, 72.70; H, 6.93; N, 11.56. Found: C, 72.12; H, 6.95; N, 11.54.

Anthracene-Linked Ditopic Receptor 10. A THF-Et ${ }_{3} \mathrm{~N}(4+1 \mathrm{~mL})$ mixed solution of 19 (1.86 g, $5.31 \mathrm{mmol}), \mathbf{2 9}^{21}$ (111 mg, $\left.0.49 \mathrm{mmol}\right)$, and $\left(\mathrm{Ph}_{3} \mathrm{P}\right)_{2} \mathrm{PdCl}_{2}(17 \mathrm{mg}$, $24 \mu \mathrm{mol}$ ) was stirred at $70{ }^{\circ} \mathrm{C}$ for $2 \mathrm{~h}$. After removal of the solvent, the residue was poured into water and extracted with $\mathrm{CH}_{2} \mathrm{Cl}_{2}$. The $\mathrm{CH}_{2} \mathrm{Cl}_{2}$ extract was evaporated and chromatographed (silica gel; eluent, $\mathrm{CH}_{2} \mathrm{Cl}_{2} / \mathrm{MeOH}$ 50:1) to give 10: yield 64\% (240 mg); mp 229-231 ${ }^{\circ} \mathrm{C}$; IR (KBr) 3299, 2207, 1676, $1552 \mathrm{~cm}^{-1} ;{ }^{1} \mathrm{H} \mathrm{NMR}\left(\mathrm{CDCl}_{3}\right) \delta 9.47$ (s, $\left.1 \mathrm{H}\right)$, $8.48(\mathrm{~s}, 1 \mathrm{H}), 8.06(\mathrm{~d}, J=8.7 \mathrm{~Hz}, 2 \mathrm{H}), 7.92(\mathrm{br} \mathrm{s}, 4 \mathrm{H}), 7.82(\mathrm{~d}, J=7.5 \mathrm{~Hz}, 2 \mathrm{H}), 7.67$ (br s, 4 H), 7.50 (dd, $J=8.7,7.1 \mathrm{~Hz}, 2 \mathrm{H}), 2.36-2.24$ (m, 8 H), 1.70-1.45 (m, 8 H), 1.44-1.29 $(\mathrm{m}, 8 \mathrm{H}), 0.98-0.86(\mathrm{~m}, 12 \mathrm{H}) ;{ }^{13} \mathrm{C} \mathrm{NMR}\left(\mathrm{CDCl}_{3}\right) \delta 171.37,149.59,136.09,131.71$, $131.55,131.47,129.95,127.79,125.35,123.99,120.71,111.92,93.17,92.49,37.45$, 27.53, 22.69, 14.13; FABMS (in 3-nitrobenzyl alcohol) $m / e$ (rel intensity) $777\left(\mathrm{MH}^{+}\right.$, $100 \%$ ). Anal. Calcd for $\mathrm{C}_{48} \mathrm{H}_{52} \mathrm{~N}_{6} \mathrm{O}_{4}: \mathrm{C}, 74.20 ; \mathrm{H}, 8.24 ; \mathrm{N}, 10.82$. Found: C, 73.90; $\mathrm{H}$, 8.06; N, 10.53 .

2,6-Di- $\boldsymbol{n}$-pentamido-4-ethynylpyridine (14). This compound was synthesized from 4-(allyloxy)-2,6-diaminopyridine ${ }^{18}$ (16) in 5 steps in a manner similar to that described for 13. ${ }^{13}$ To a $\mathrm{CH}_{2} \mathrm{Cl}_{2}(140 \mathrm{~mL})$ solution of $16(2.3 \mathrm{~g}, 13.9 \mathrm{mmol})$ and $\mathrm{Et}_{3} \mathrm{~N}$ $(5.3 \mathrm{~mL})$ was added $n$-valeryl chloride $(3.7 \mathrm{~g}, 30.6 \mathrm{mmol})$ dropwise at $0{ }^{\circ} \mathrm{C}$. The reaction mixture was stirred at room temperature for $5 \mathrm{~h}$, washed with water, and evaporated. The residue was subjected to column chromatography (silica gel; eluent, $\mathrm{CHCl}_{3} / \mathrm{AcOEt}$ 50:1) to give 4-(allyloxy)-2,6-di- $n$-pentamidopyridine (17): yield 93\% (4.3 g); mp 47-49 ${ }^{\circ} \mathrm{C}$; IR (KBr) $3421,1687,1585 \mathrm{~cm}^{-1} ;{ }^{1} \mathrm{H}$ NMR $\left(\mathrm{CDCl}_{3}\right) \delta 7.57$ (br s, $\left.4 \mathrm{H}\right), 6.07-5.96(\mathrm{~m}, 1 \mathrm{H})$, $5.43(\mathrm{dd}, J=17.2,1.3 \mathrm{~Hz}, 1 \mathrm{H}), 5.30(\mathrm{dd}, J=10.6,1.3 \mathrm{~Hz}, 1 \mathrm{H}), 4.61(\mathrm{dd}, J=5.4,1.5 \mathrm{~Hz}$, $2 \mathrm{H}), 2.35(\mathrm{t}, J=7.3 \mathrm{~Hz}, 4 \mathrm{H}), 1.74-1.65(\mathrm{~m}, 4 \mathrm{H}), 1.45-1.34(\mathrm{~m}, 4 \mathrm{H}), 0.94(\mathrm{t}, J=7.3 \mathrm{~Hz}$, 
$6 \mathrm{H}) ;{ }^{13} \mathrm{C} \mathrm{NMR}\left(\mathrm{CDCl}_{3}\right) \delta 171.67,168.47,150.51,132.05,118.32,96.37,69.03,37.61$, 27.37, 22.31, 13.79; FABMS (in 3-nitrobenzyl alcohol) $m / e$ (rel intensity) $334\left(\mathrm{MH}^{+}\right.$, $100 \%$ ). Anal. Calcd for $\mathrm{C}_{18} \mathrm{H}_{27} \mathrm{~N}_{3} \mathrm{O}_{3}$ : C, 64.84; H, 8.16; N, 12.60. Found: C, 64.58; H, 8.20; $\mathrm{N}, 12.38 . \quad \mathrm{A} \mathrm{CH}_{3} \mathrm{CN}-\mathrm{EtOH}-\mathrm{H}_{2} \mathrm{O}(15+15+15 \mathrm{~mL})$ mixed solution of $\mathbf{1 7}(2.3 \mathrm{~g}, 90$ $\mathrm{mmol}),\left(\mathrm{Ph}_{3} \mathrm{P}\right)_{3} \mathrm{RhCl}(511 \mathrm{mg}, 0.55 \mathrm{mmol})$, and DABCO (77 mg, $\left.0.69 \mathrm{mmol}\right)$ was stirred at $70{ }^{\circ} \mathrm{C}$ for $18 \mathrm{~h}$. After removal of the solvent, the residue was subjected to column chromatography (silica gel; eluent, $\mathrm{CH}_{2} \mathrm{Cl}_{2} / \mathrm{MeOH}$ 20:1) to give 2,6-di- $n$-pentamido-4pyridone (18): yield 79\% (1.6 g); mp $168-169^{\circ} \mathrm{C}$; IR (KBr) $1662 \mathrm{~cm}^{-1}$; ${ }^{1} \mathrm{H}$ NMR (DMSO$\left.d_{6}\right) \delta 10.47(\mathrm{~s}, 1 \mathrm{H}), 9.74(\mathrm{~s}, 2 \mathrm{H}), 7.25(\mathrm{~s}, 2 \mathrm{H}), 2.34(\mathrm{t}, J=7.3 \mathrm{~Hz}, 4 \mathrm{H}), 1.57-1.48(\mathrm{~m}, 4$ $\mathrm{H}), 1.34-1.22(\mathrm{~m}, 4 \mathrm{H}), 0.87(\mathrm{t}, J=7.3 \mathrm{~Hz}, 6 \mathrm{H}) ;{ }^{13} \mathrm{C}$ NMR (DMSO- $\left.d_{6}\right) \delta 172.07,166.55$, 151.24, 96.84, 35.86, 27.18, 21.73, 13.72; FABMS (in 3-nitrobenzyl alcohol) m/e (rel intensity) $294\left(\mathrm{MH}^{+}, 100 \%\right)$. Anal. Calcd for $\mathrm{C}_{15} \mathrm{H}_{23} \mathrm{~N}_{3} \mathrm{O}_{3}: \mathrm{C}, 61.41 ; \mathrm{H}, 7.90 ; \mathrm{N}, 14.32$. Found: C, 60.84; H, 7.90; N, 14.27. To a pyridine $(10 \mathrm{~mL})$ solution of $\mathbf{1 8}(2.3 \mathrm{~g}, 7.84$ mmol) was added trifluoromethanesulfonic anhydride $(2.9 \mathrm{~g}, 10.2 \mathrm{mmol})$ dropwise at $0{ }^{\circ} \mathrm{C}$ over a $5 \mathrm{~min}$ period. The reaction mixture was stirred at room temperature for $5 \mathrm{~h}$. After removal of the solvent, the residue was poured into water and extracted with ether. The ether extract was evaporated and chromatographed (silica gel; eluent, $\mathrm{CH}_{2} \mathrm{Cl}_{2} / \mathrm{MeOH}$ 30:1) to give 4-(2,6-di- $n$-pentamidopyridyl) trifluoromethanesulfonate (19): yield 87\% (2.8 g); mp 72-73 ${ }^{\circ} \mathrm{C}$; IR (KBr) 3396, 1672, $1217 \mathrm{~cm}^{-1} ;{ }^{1} \mathrm{H} \mathrm{NMR}\left(\mathrm{CDCl}_{3}\right) \delta 7.95$ (s, $\left.2 \mathrm{H}\right)$, $7.72(\mathrm{~s}, 2 \mathrm{H}), 2.39$ (t, $J=7.6 \mathrm{~Hz}, 4 \mathrm{H}), 1.76-1.63(\mathrm{~m}, 4 \mathrm{H}), 1.47-1.33(\mathrm{~m}, 4 \mathrm{H}), 0.95$ (t, $J$ $=7.3 \mathrm{~Hz}, 6 \mathrm{H}) ;{ }^{13} \mathrm{C} \mathrm{NMR}\left(\mathrm{CDCl}_{3}\right) \delta 171.82,158.65,151.12,118.61\left(\mathrm{q}, J_{\mathrm{C}-\mathrm{F}}=321 \mathrm{~Hz}\right)$, 102.14, 37.42, 27.11, 22.26, 13.76; FABMS (in 3-nitrobenzyl alcohol) $m / e$ (rel intensity) $426\left(\mathrm{MH}^{+}, 100 \%\right)$. Anal. Calcd for $\mathrm{C}_{16} \mathrm{H}_{22} \mathrm{~F}_{3} \mathrm{~N}_{3} \mathrm{O}_{5} \mathrm{~S}: \mathrm{C}, 45.17 ; \mathrm{H}, 5.21 ; \mathrm{N}, 9.88$. Found: $\mathrm{C}$, 45.03; H, 5.10; N, 9.84. To an $\mathrm{Et}_{3} \mathrm{~N}(18 \mathrm{~mL})$ solution of 19 (1.7 g, $\left.4.13 \mathrm{mmol}\right)$ and $\left(\mathrm{Ph}_{3} \mathrm{P}\right)_{2} \mathrm{PdCl}_{2}(58 \mathrm{mg}, 83 \mu \mathrm{mol})$ was added (trimethylsilyl)acetylene $(811 \mathrm{mg}, 8.26 \mathrm{mmol})$ at $40{ }^{\circ} \mathrm{C}$. The reaction mixture was stirred at $70{ }^{\circ} \mathrm{C}$ for $2 \mathrm{~h}$. After removal of the solvent, the residue was poured into water and extracted with $\mathrm{CH}_{2} \mathrm{Cl}_{2}$. The $\mathrm{CH}_{2} \mathrm{Cl}_{2}$ extract was evaporated and chromatographed (silica gel; eluent, $\mathrm{CH}_{2} \mathrm{Cl}_{2}$ ) to give 2,6-di-npentamido-4-[(trimethylsilyl)ethynyl]pyridine (20): yield 94\% (1.5 g); mp $126-128{ }^{\circ} \mathrm{C}$; 
IR (KBr) 3423, 2164, 1699, 1556, 849, $764 \mathrm{~cm}^{-1} ;{ }^{1} \mathrm{H}$ NMR $\left(\mathrm{CDCl}_{3}\right) \delta 7.98(\mathrm{~s}, 2 \mathrm{H}), 7.50$ $(\mathrm{s}, 2 \mathrm{H}), 2.37(\mathrm{t}, J=7.6 \mathrm{~Hz}, 4 \mathrm{H}), 1.75-1.66(\mathrm{~m}, 4 \mathrm{H}), 1.46-1.35(\mathrm{~m}, 4 \mathrm{H}), 0.95(\mathrm{t}, J=7.3$ $\mathrm{Hz}, 6 \mathrm{H}), 0.24(\mathrm{~s}, 3 \mathrm{H}) ;{ }^{13} \mathrm{C} \mathrm{NMR}\left(\mathrm{CDCl}_{3}\right) \delta 171.68,149.45,135.53,111.70,102.25$, 99.56, 37.26, 27.24, 22.17, 13.68, -0.48; FABMS (in 3-nitrobenzyl alcohol) m/e (rel intensity) $374\left(\mathrm{MH}^{+}, 100 \%\right)$. Anal. Calcd for $\mathrm{C}_{20} \mathrm{H}_{31} \mathrm{~N}_{3} \mathrm{O}_{2} \mathrm{Si}: \mathrm{C}, 64.30 ; \mathrm{H}, 8.36 ; \mathrm{N}, 11.25$. Found: C, 64.31; H, 8.25; N, 11.13. To a THF (30 mL) solution of $\mathbf{2 0}(1.3 \mathrm{~g}, 3.53 \mathrm{mmol})$ was added tetra- $n$-butylammonium fluoride $(1.4 \mathrm{mg}, 5.30 \mathrm{mmol})$ and a few drops of water at room temperature. The reaction mixture was stirred at the same temperature for $1.5 \mathrm{~h}$. After removal of the solvent, the residue was poured into water and extract with $\mathrm{CH}_{2} \mathrm{Cl}_{2}$. The $\mathrm{CH}_{2} \mathrm{Cl}_{2}$ extract was evaporated and chromatographed (silica gel; eluent, $\mathrm{CH}_{2} \mathrm{Cl}_{2}$ ) to give 14: yield $96 \%$ (1.0 g); $\mathrm{mp} 135-136{ }^{\circ} \mathrm{C}$; IR (KBr) 3423, 3255, 2104, 1699, $1560 \mathrm{~cm}^{-1}$; ${ }^{1} \mathrm{H} \mathrm{NMR}\left(\mathrm{CDCl}_{3}\right) \delta 8.01(\mathrm{~s}, 2 \mathrm{H}), 7.54($ br s, $2 \mathrm{H}), 3.24(\mathrm{~s}, 1 \mathrm{H}), 2.37(\mathrm{t}, J=7.3 \mathrm{~Hz}, 4 \mathrm{H})$, 1.75-1.65 (m, $4 \mathrm{H}), 1.46-1.35(\mathrm{~m}, 4 \mathrm{H}), 0.95(\mathrm{t}, J=7.3 \mathrm{~Hz}, 6 \mathrm{H}) ;{ }^{13} \mathrm{C} \mathrm{NMR}\left(\mathrm{CDCl}_{3}\right) \delta$ $171.51,149.51,134.81,112.02,81.49,81.37,37.55,27.36,22.30,13.79$; FABMS (in 3nitrobenzyl alcohol) $m / e$ (rel intensity) $302\left(\mathrm{MH}^{+}, 100 \%\right)$. Anal. Calcd for $\mathrm{C}_{17} \mathrm{H}_{23} \mathrm{~N}_{3} \mathrm{O}_{2}: \mathrm{C}$, 67.75; H, 7.69; N, 13.94. Found: C, 68.15; H, 7.80; N, 13.91.

1-n-Octyluracil (22). To a DMSO (100 mL) suspension of uracil (21: $10 \mathrm{~g}, 89$ mmol) and finely ground $\mathrm{K}_{2} \mathrm{CO}_{3}(62 \mathrm{~g}, 450 \mathrm{mmol})$ was added a DMSO (50 mL) solution of 1-bromooctane $(21 \mathrm{~g}, 110 \mathrm{mmol})$ dropwise at $0{ }^{\circ} \mathrm{C}$. The reaction mixture was stirred at room temperature for $12 \mathrm{~h}$ and filtered. The filtrate was evaporated, and the residue was poured into water and extracted with $\mathrm{CHCl}_{3}$ (ca. $200 \mathrm{~mL}$ ). The $\mathrm{CHCl}_{3}$ extract was concentrated to one-tenth of its original volume and dropped into a large quantity of cold $\left(\right.$ ca. $0{ }^{\circ} \mathrm{C}$ ) hexane with stirring. The resulting precipitate was filtered and washed with hexane to give 22: yield 39\% (7.8 g); mp 69-70 ${ }^{\circ} \mathrm{C}$; IR (KBr) $1693,1649 \mathrm{~cm}^{-1}$; ${ }^{1} \mathrm{H}$ NMR $\left(\mathrm{CDCl}_{3}\right) \delta 9.54(\mathrm{br} \mathrm{s}, 1 \mathrm{H}), 7.16(\mathrm{~d}, J=7.8 \mathrm{~Hz}, 1 \mathrm{H}), 5.71(\mathrm{~d}, J=7.8 \mathrm{~Hz}, 1 \mathrm{H}), 3.72(\mathrm{t}, J=$ $7.3 \mathrm{~Hz}, 2 \mathrm{H}), 1.77-1.60(\mathrm{~m}, 2 \mathrm{H}), 1.40-1.20(\mathrm{~m}, 10 \mathrm{H}), 0.88(\mathrm{t}, J=6.8 \mathrm{~Hz}, 3 \mathrm{H}) ;{ }^{13} \mathrm{C} \mathrm{NMR}$ $\left(\mathrm{CDCl}_{3}\right) \delta 163.94,150.90,144.41,102.03,48.86,31.66,29.07,29.04,29.00,26.36$, 22.54, 14.01; FABMS (in 3-nitrobenzyl alcohol) $m / e$ (rel intensity) $225\left(\mathrm{MH}^{+}, 100 \%\right)$. 
Anal. Calcd for $\mathrm{C}_{12} \mathrm{H}_{20} \mathrm{~N}_{2} \mathrm{O}_{2}$ : C, 64.26; H, 8.99; N, 12.49. Found: C, 64.08; H, 9.04; N, 12.56 .

5-Iodo-1-n-octyluracil (23). To a MeOH (30 mL) solution of $22(5.0 \mathrm{~g}, 22$ mmol) was added $\mathrm{ICl}(7.0 \mathrm{~g}, 44 \mathrm{mmol})$ dropwise at room temperature. The reaction mixture was stirred at the same temperature for $10 \mathrm{~h}$ and cooled to $-78{ }^{\circ} \mathrm{C}$. The resulting precipitate was filtered and washed with cold (ca. $0{ }^{\circ} \mathrm{C}$ ) hexane to give 23 : yield $75 \%(5.8$ g); mp 152-154 ${ }^{\circ} \mathrm{C}$; IR (KBr) 1703, $1674 \mathrm{~cm}^{-1} ;{ }^{1} \mathrm{H}$ NMR $\left(\mathrm{CDCl}_{3}\right) \delta 8.83(\mathrm{~s}, 1 \mathrm{H}), 7.61$ (s, $1 \mathrm{H}), 3.74(\mathrm{t}, J=7.3 \mathrm{~Hz}, 2 \mathrm{H}), 1.75-1.62(\mathrm{~m}, 2 \mathrm{H}), 1.41-1.20(\mathrm{~m}, 10 \mathrm{H}), 6.88(\mathrm{t}, J=6.5$ $\mathrm{Hz}, 3 \mathrm{H}) ;{ }^{13} \mathrm{C} \mathrm{NMR}\left(\mathrm{CDCl}_{3}\right) \delta 160.33,150.30,148.87,67.45,49.23,31.68,29.17,29.04$, 26.31, 22.58, 14.05; FABMS (in 3-nitrobenzyl alcohol) m/e (rel intensity) $351\left(\mathrm{MH}^{+}\right.$, $100 \%$ ). Anal. Calcd for $\mathrm{C}_{12} \mathrm{H}_{19} \mathrm{IN}_{2} \mathrm{O}_{2}$ : C, 41.16; H, 5.47; N, 8.00. Found: C, 41.84; H, 5.48; $\mathrm{N}, 8.18$.

1-n-Octyl-5-[(trimethylsilyl)ethynyl]uracil (24). To an $\mathrm{Et}_{3} \mathrm{~N}(140 \mathrm{~mL})$ solution of 23 (5.0 g, $14.3 \mathrm{mmol}),\left(\mathrm{Ph}_{3} \mathrm{P}\right)_{2} \mathrm{PdCl}_{2}(200 \mathrm{mg}, 0.29 \mathrm{mmol})$, and $\mathrm{CuI}(27 \mathrm{mg}$, $0.14 \mathrm{mmol}$ ) was added (trimethylsilyl)acetylene $(5.6 \mathrm{~g}, 57 \mathrm{mmol})$ dropwise at $40{ }^{\circ} \mathrm{C}$. The reaction mixture was stirred at $60{ }^{\circ} \mathrm{C}$ for $2 \mathrm{~h}$ and evaporated. The residue was poured into water and extracted with $\mathrm{CH}_{2} \mathrm{Cl}_{2}$. The $\mathrm{CH}_{2} \mathrm{Cl}_{2}$ extract was evaporated and chromatographed (silica gel; eluent, $\mathrm{CHCl}_{3} / \mathrm{AcOEt} 15: 1$ ) to give 24: yield 85\% (3.9 g); mp 94-95 ${ }^{\circ} \mathrm{C}$; IR (KBr) 3419, 2158, 1713, $1678 \mathrm{~cm}^{-1} ;{ }^{1} \mathrm{H}$ NMR $\left(\mathrm{CDCl}_{3}\right) \delta 9.06(\mathrm{br} \mathrm{s}, 1 \mathrm{H})$, $7.47(\mathrm{~s}, 1 \mathrm{H}), 3.73(\mathrm{t}, J=7.3 \mathrm{~Hz}, 2 \mathrm{H}), 1.74-1.64(\mathrm{~m}, 2 \mathrm{H}), 1.36-1.21(\mathrm{~m}, 10 \mathrm{H}), 0.88$ (t, $J$ $=6.7 \mathrm{~Hz}, 3 \mathrm{H}), 0.23(\mathrm{~s}, 9 \mathrm{H}) ;{ }^{13} \mathrm{C} \mathrm{NMR}\left(\mathrm{CDCl}_{3}\right) \delta 161.62,149.67,147.68,99.89,99.65$, 94.99, 49.29, 31.66, 29.07, 29.04, 26.30, 22.56, 14.04, -0.19; FABMS (in 3-nitrobenzyl alcohol) m/e (rel intensity) $321\left(\mathrm{MH}^{+}, 100 \%\right)$. Anal. Calcd for $\mathrm{C}_{17} \mathrm{H}_{28} \mathrm{~N}_{2} \mathrm{O}_{2} \mathrm{Si}: \mathrm{C}, 63.71 ; \mathrm{H}$, 8.81; N, 8.74. Found: C, 63.66; H, 8.85; N, 8.84.

5-Ethynyl-1-n-octyluracil (25). To a THF (100 mL) solution of $24(3.9 \mathrm{~g}, 12.2$ mmol) was added tetra- $n$-butylammonium fluoride $(4.8 \mathrm{~g}, 18.3 \mathrm{mmol})$ and a few drops of 
water at room temperature. The reaction mixture was stirred at the same temperature for $1.5 \mathrm{~h}$. After removal of the solvent, the residue was chromatographed (silica gel; eluent, $\left.\mathrm{CHCl}_{3} / \mathrm{AcOEt} 10: 1\right)$ to give 25: yield 69\% (2.1 g); mp 116-118 ${ }^{\circ} \mathrm{C}$; IR (KBr) 3242, 2115 , $1705,1672 \mathrm{~cm}^{-1} ;{ }^{1} \mathrm{H}$ NMR $\left(\mathrm{CDCl}_{3}\right) \delta 8.40(\mathrm{~s}, 1 \mathrm{H}), 7.50(\mathrm{~s}, 1 \mathrm{H}), 3.78-3.67(\mathrm{~m}, 2 \mathrm{H}), 3.21$ $(\mathrm{s}, 1 \mathrm{H}), 1.78-1.64(\mathrm{~m}, 2 \mathrm{H}), 1.41-1.20(\mathrm{~m}, 10 \mathrm{H}), 1.00-0.75(\mathrm{~m}, 3 \mathrm{H}) ;{ }^{13} \mathrm{C} \mathrm{NMR}\left(\mathrm{CDCl}_{3}\right)$ $\delta 162.06,149.81,148.08,98.64,82.08,74.41,49.35,31.62,28.99,26.26,22.51,13.98$; FABMS (in 3-nitrobenzyl alcohol) $m / e$ (rel intensity) $249\left(\mathrm{MH}^{+}, 100 \%\right)$. Anal. Calcd for $\mathrm{C}_{14} \mathrm{H}_{20} \mathrm{~N}_{2} \mathrm{O}_{2}: \mathrm{C}, 67.71 ; \mathrm{H}, 8.12 ; \mathrm{N}, 11.28$. Found: C, 67.73; H, 8.01; N, 11.31 .

1,8-Diiodobiphenylene (28). This compound was synthesized from 1,8dibromobiphenylene ${ }^{20}(\mathbf{2 7})$ by a modification of our published procedure ${ }^{25}$ in which DMF was used as a solvent instead of carcinogenic HMPA. Indeed, most iodoarenes may satisfactorily be prepared from the corresponding bromoarenes by this modified method. Details will be reported elsewhere. A DMF (30 mL) suspension of $27(800 \mathrm{mg}, 2.58$ $\mathrm{mmol}), \mathrm{CuI}(7.7 \mathrm{~g}, 39 \mathrm{mmol})$, and $\mathrm{KI}(16 \mathrm{~g}, 97 \mathrm{mmol})$ was stirred at $140{ }^{\circ} \mathrm{C}$ for $22 \mathrm{~h}$. The reaction mixture was filtered, and the filtrate was evaporated. The residue was poured into water and extracted with $\mathrm{CH}_{2} \mathrm{Cl}_{2}$. The $\mathrm{CH}_{2} \mathrm{Cl}_{2}$ extract was evaporated to give 28: yield 92\% (963 mg); mp 181-183 ${ }^{\circ} \mathrm{C} ;{ }^{1} \mathrm{H}$ NMR $\left(\mathrm{CDCl}_{3}\right) \delta 7.03(\mathrm{~d}, J=8.5 \mathrm{~Hz}, 2 \mathrm{H}), 6.59$ $(\mathrm{d}, J=6.8 \mathrm{~Hz}, 2 \mathrm{H}), 6.48(\mathrm{dd}, J=8.5,6.8 \mathrm{~Hz}, 2 \mathrm{H}) ;{ }^{13} \mathrm{C} \mathrm{NMR}\left(\mathrm{CDCl}_{3}\right) \delta 154.89,151.79$, 139.03, 129.98, 116.31, 80.51; FABMS (in 3-nitrobenzyl alcohol) m/e (rel intensity) 404 $\left(\mathrm{M}^{+}, 58 \%\right)$. Anal. Calcd for $\mathrm{C}_{12} \mathrm{H}_{6} \mathrm{I}_{2}: \mathrm{C}, 35.68 ; \mathrm{H}, 1.50$. Found: $\mathrm{C}, 35.59 ; \mathrm{H}, 1.55$.

\section{Reference}

(13) Inouye, M.; Hyodo, Y.; Nakazumi, H. J. Org. Chem. 1999, 64, 2704-2710. Inouye, M.; Itoh, M. S.; Nakazumi, H. J. Org. Chem. 1999, 64, 9393-9398.

(18) Markees, D. G.; Dewey, V. C.; Kidder, G. W. J. Med. Chem. 1968, 11, 126-129.

(19) Bossenbroek, B.; Sanders, D. C.; Curry, H. M.; Shechter, H. J. Am. Chem. Soc. 1969, $91,371-379$.

(20) Rajca, A.; Safronov, A.; Rajca, S.; Ross, C. R., II; Stezowski, J. J. J. Am. Chem. 
Soc. 1996, 118, 7272-7279. Iyoda, M.; Humayun Kabir, S. M.; Vorasingha, A.; Kuwatani, Y.; Yoshida, M. Tetrahedron Lett. 1998, 39, 5393-5396.

(21) Katz, H. E. J. Org. Chem. 1989, 54, 2179-2183.

(23) Ogilvie, K. K. Can. J. Chem. 1973, 51, 3799-3807. Ogilvie, K. K.; Iwacha, D. J. Tetahedron Lett. 1973, 317-319. Ogilvie, K. K.; Thompson, E. A.; Quilliam, M. A.; Westmore, J. B. Tetahedron Lett. 1974, 2865-2868.

(25) Suzuki, H.; Kondo, A.; Inouye, M.; Ogawa, T. Synthesis 1986, 121-122. 
${ }^{1} \mathrm{H}$ NMR spectra of all new compounds

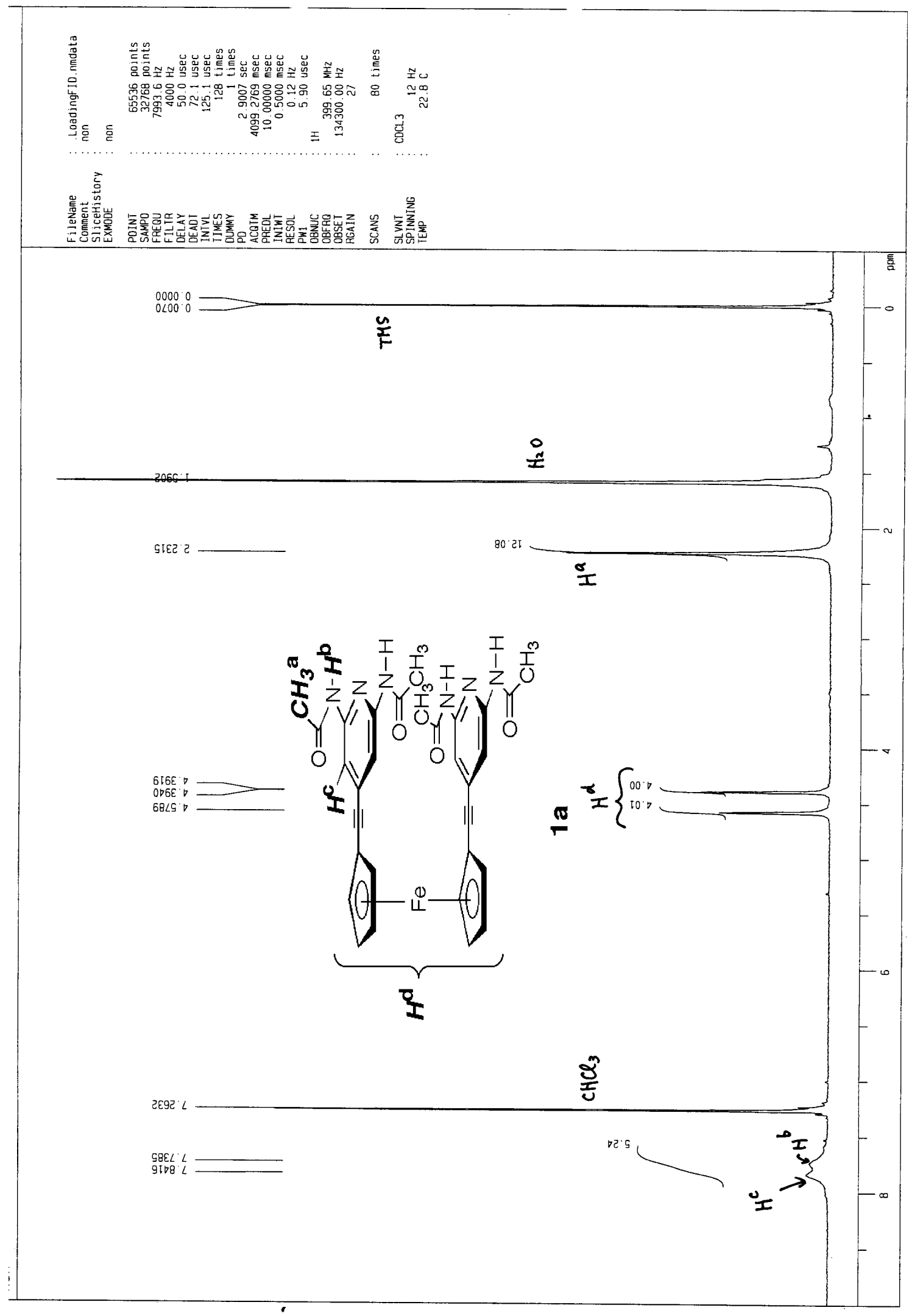




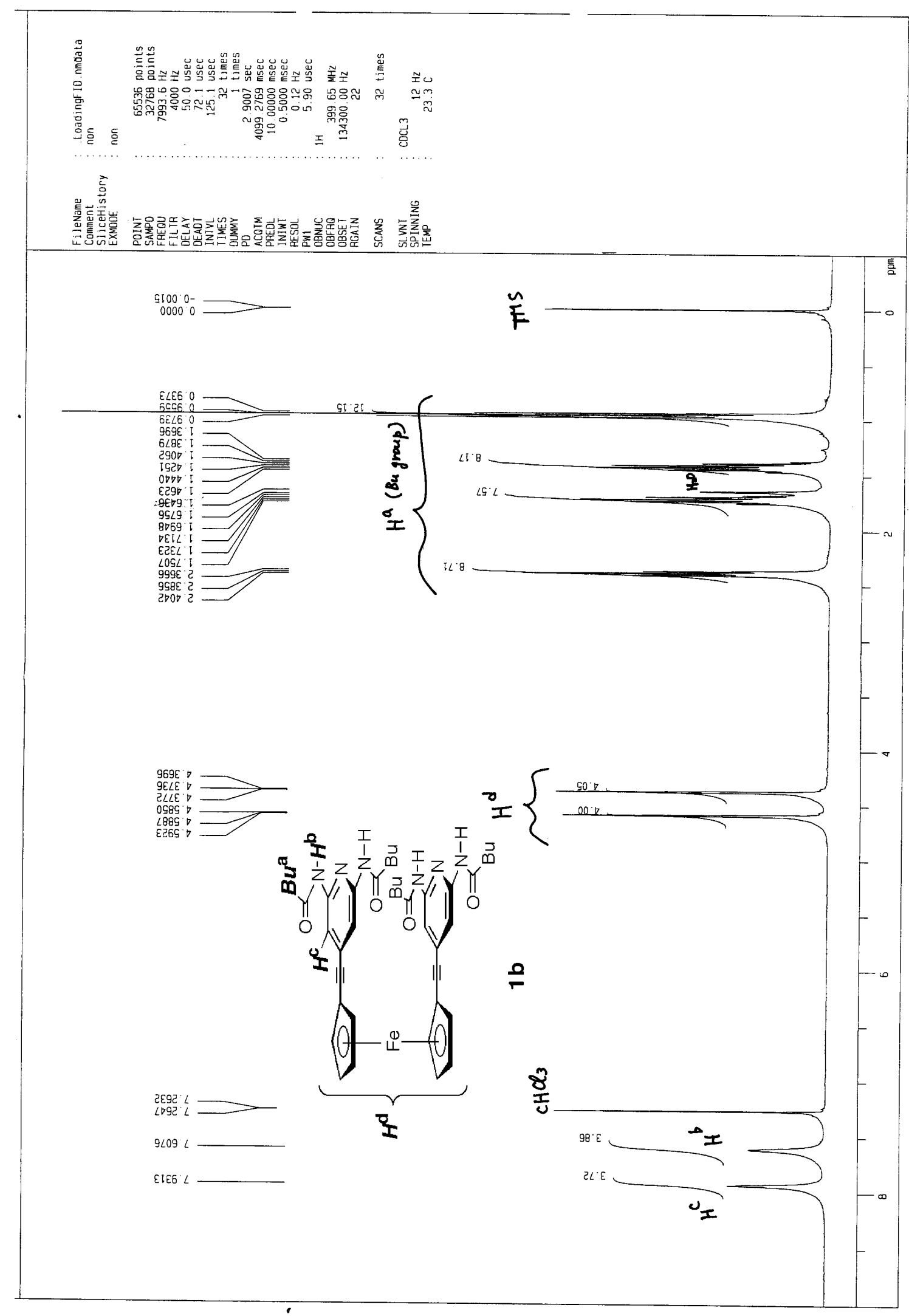




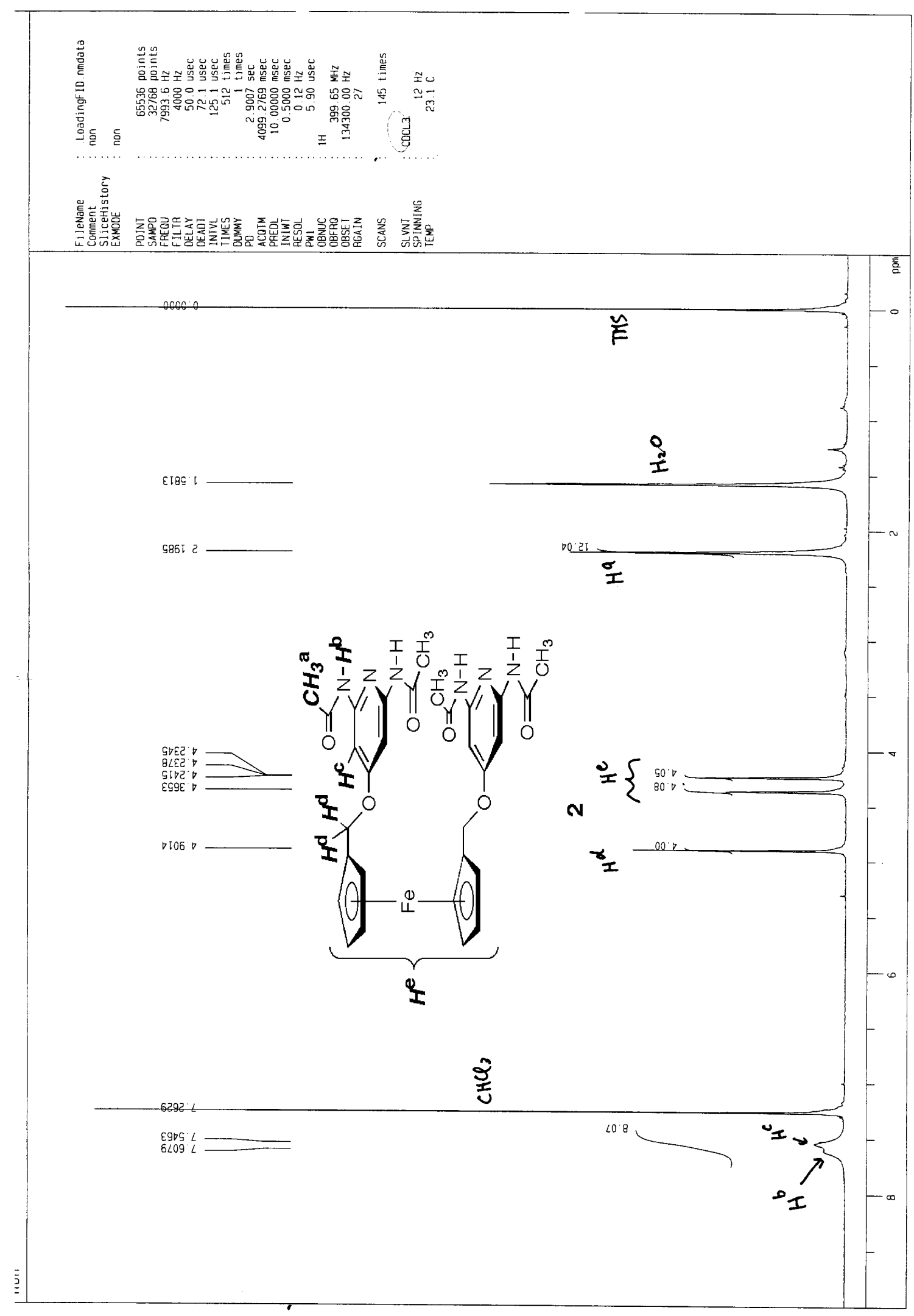




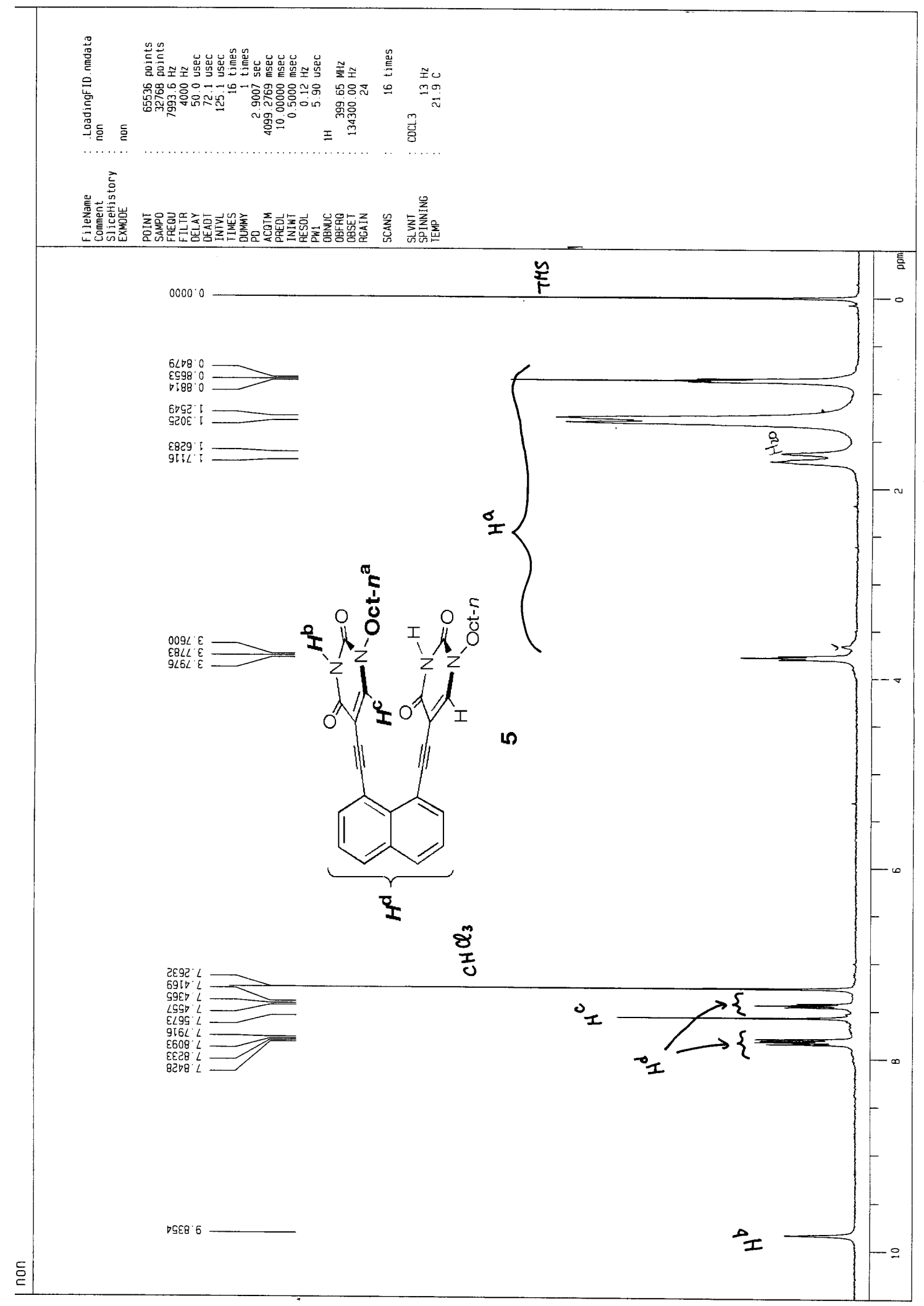




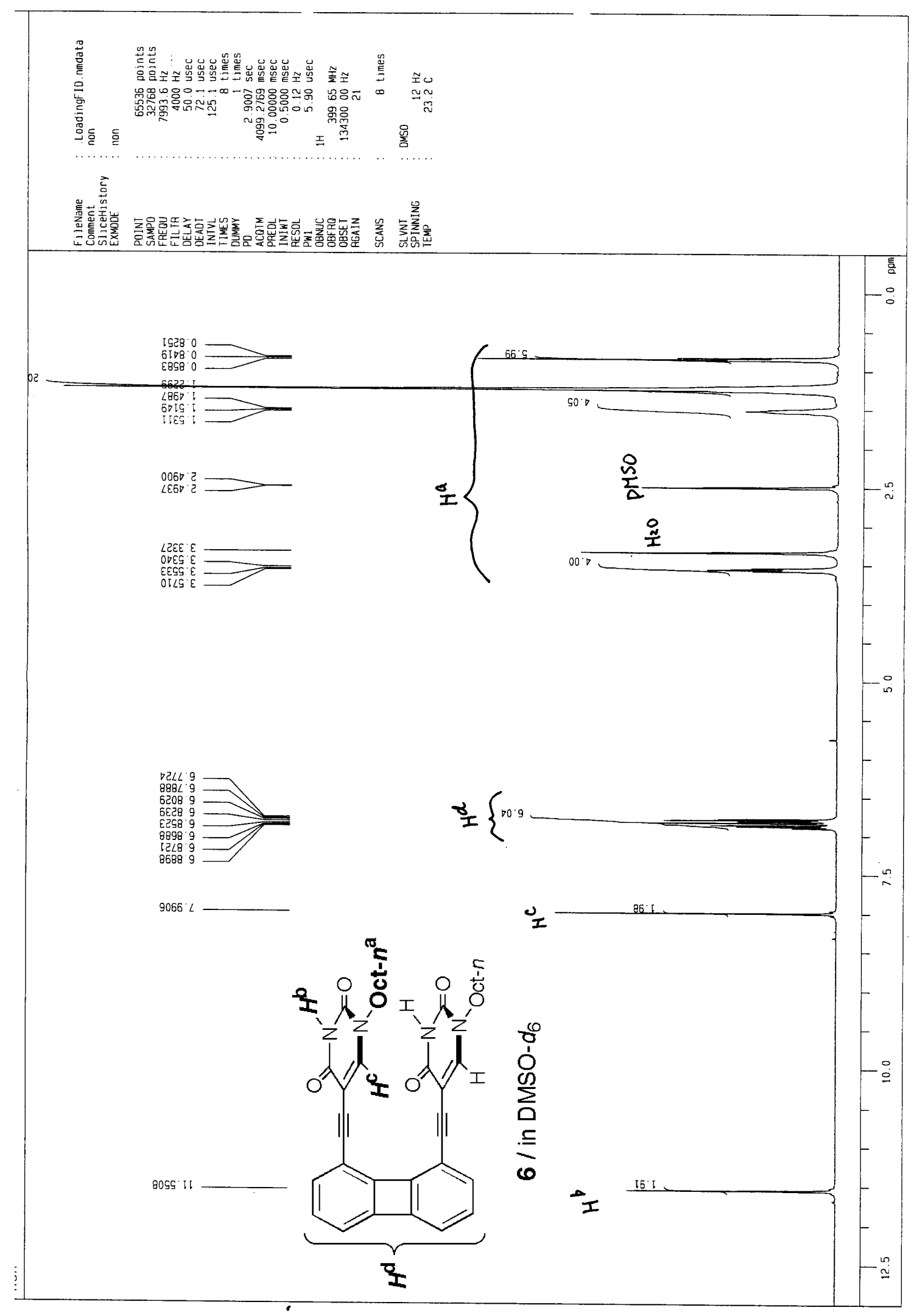




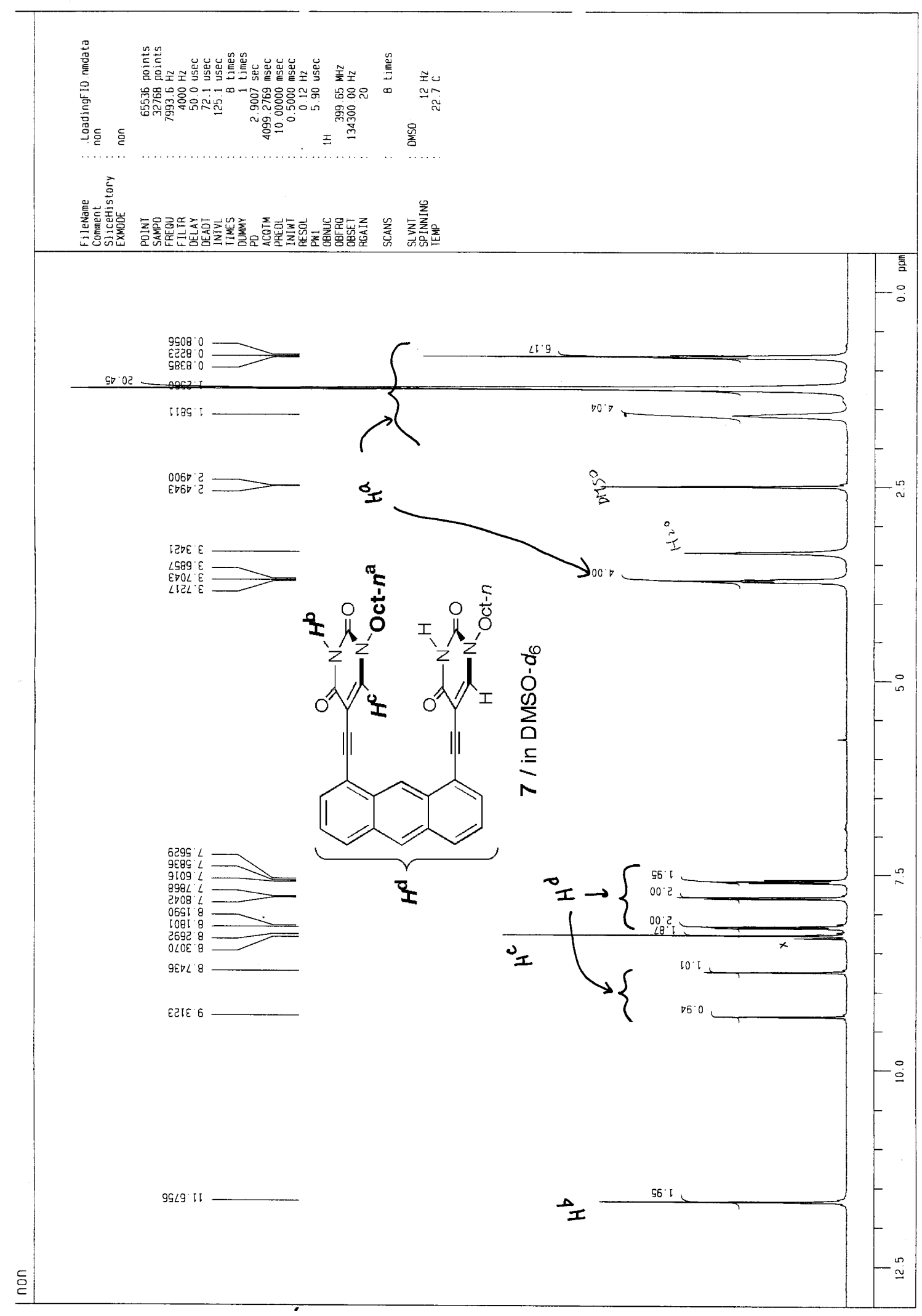




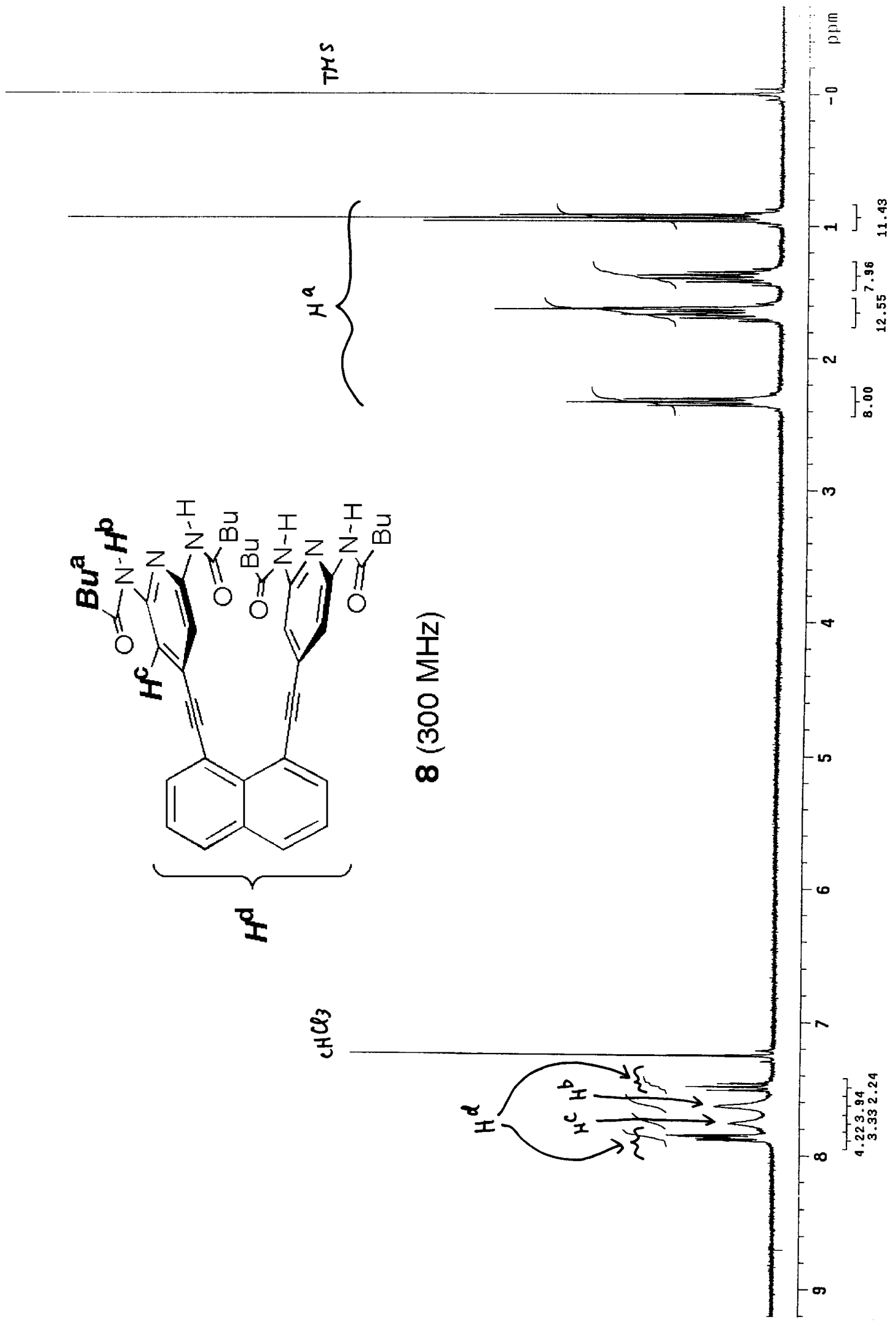




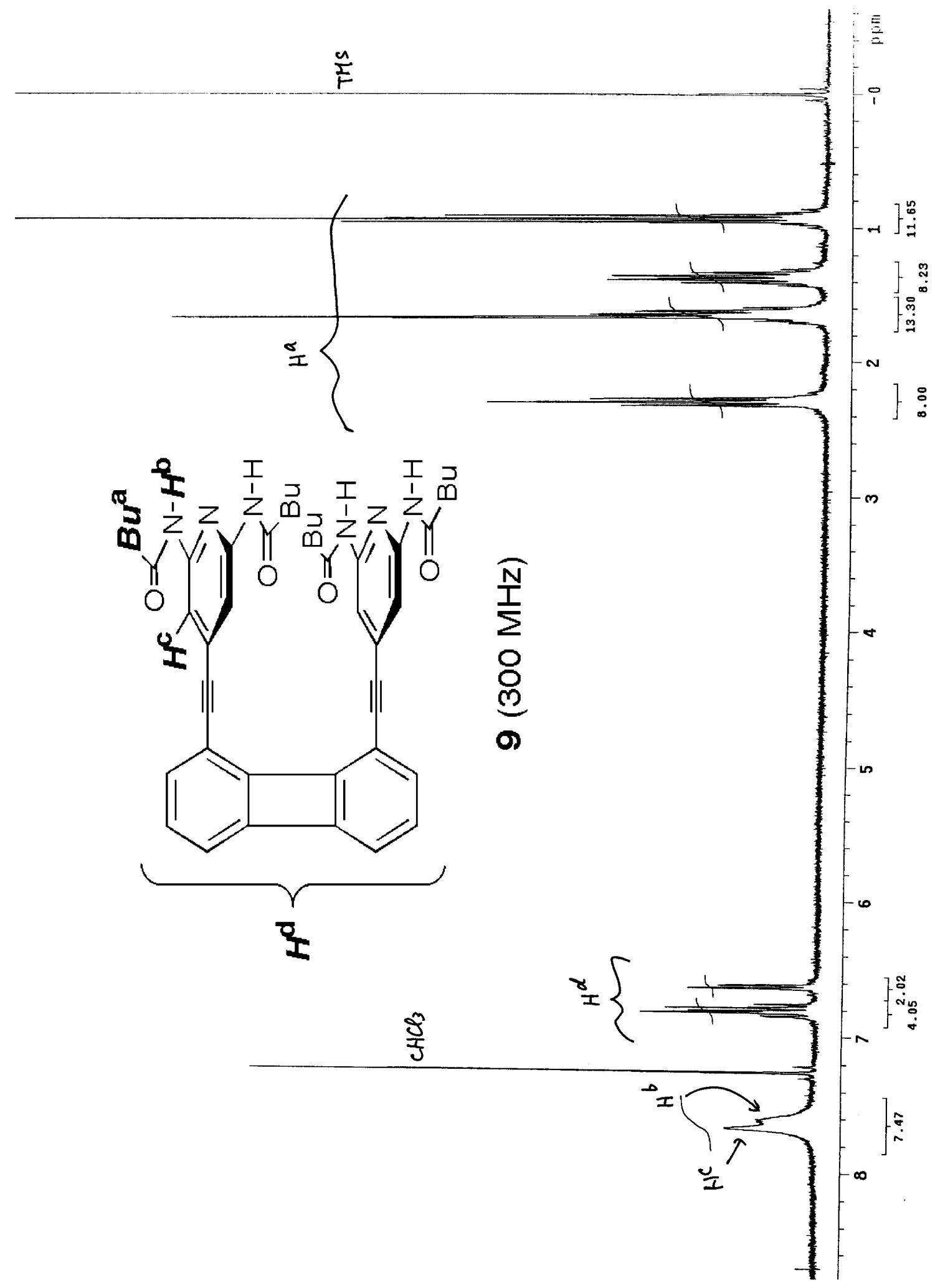




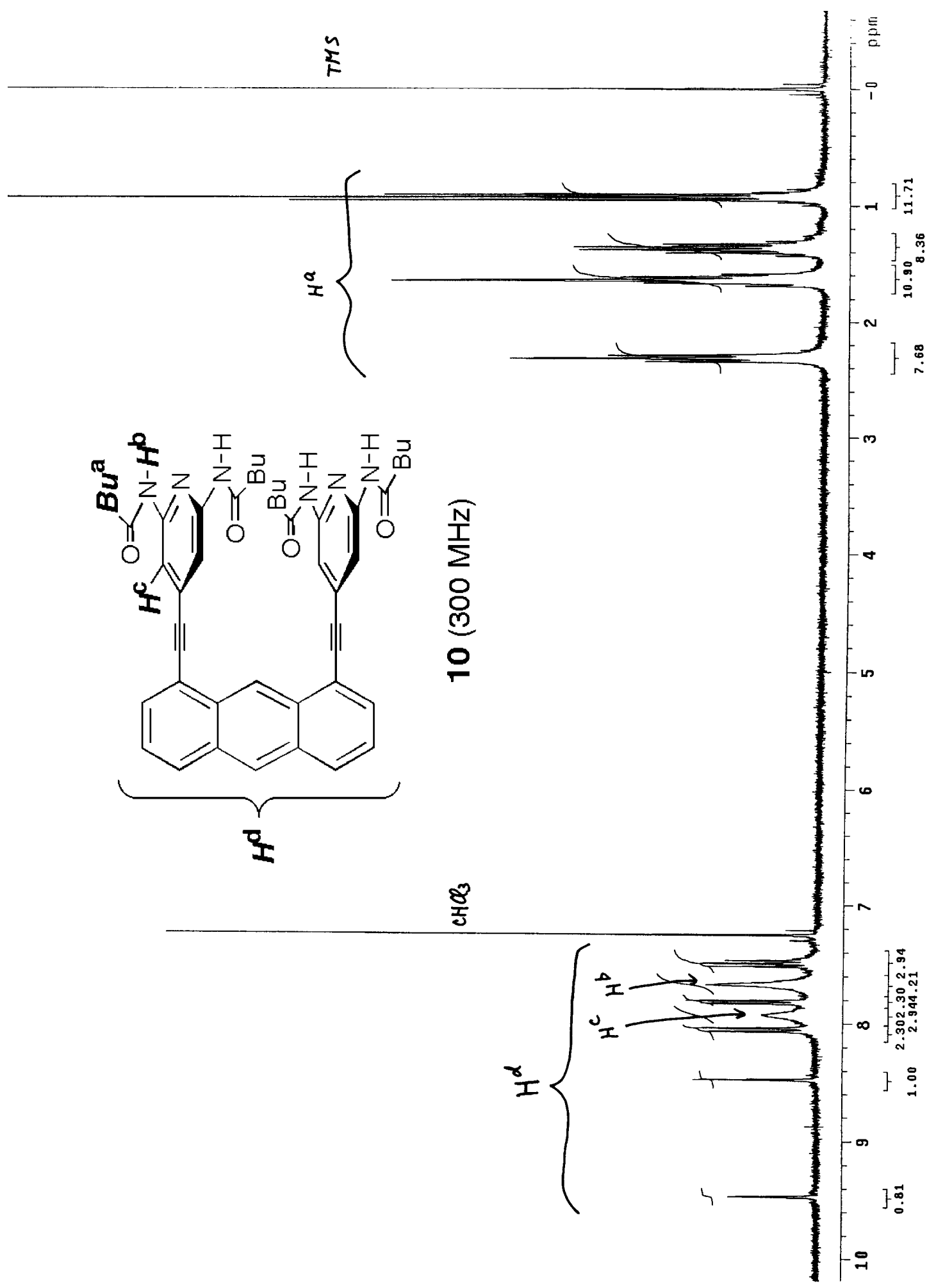




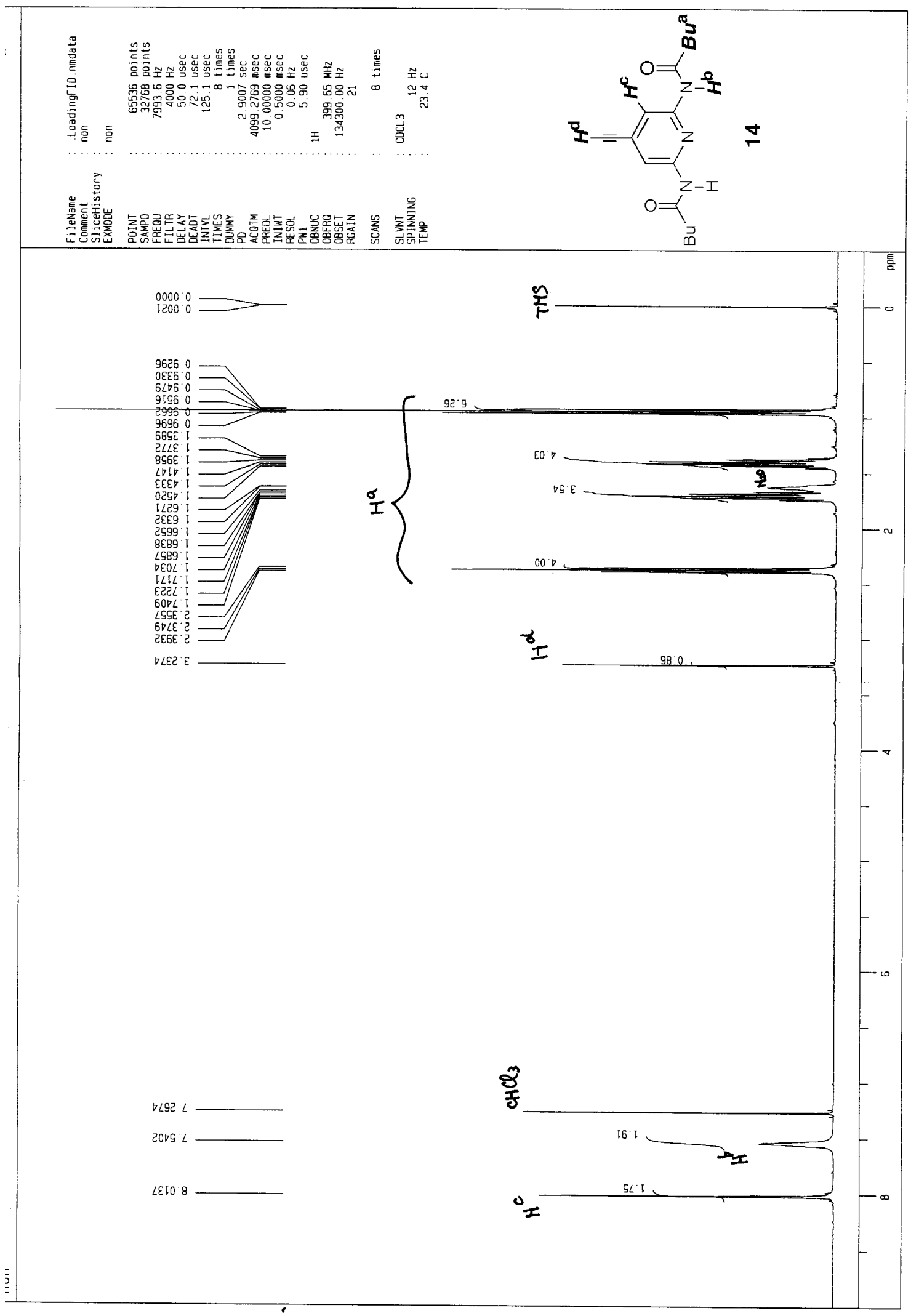




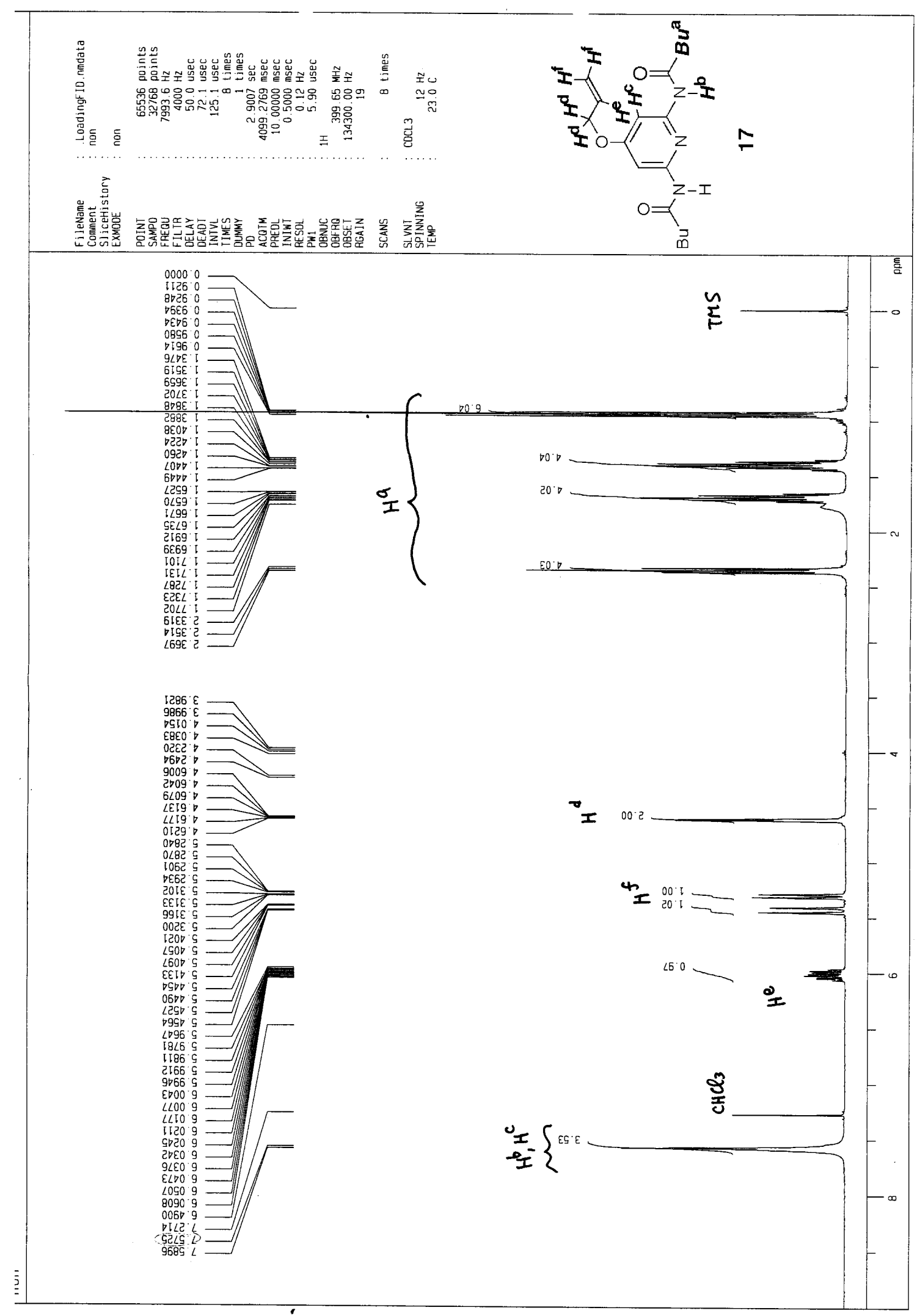




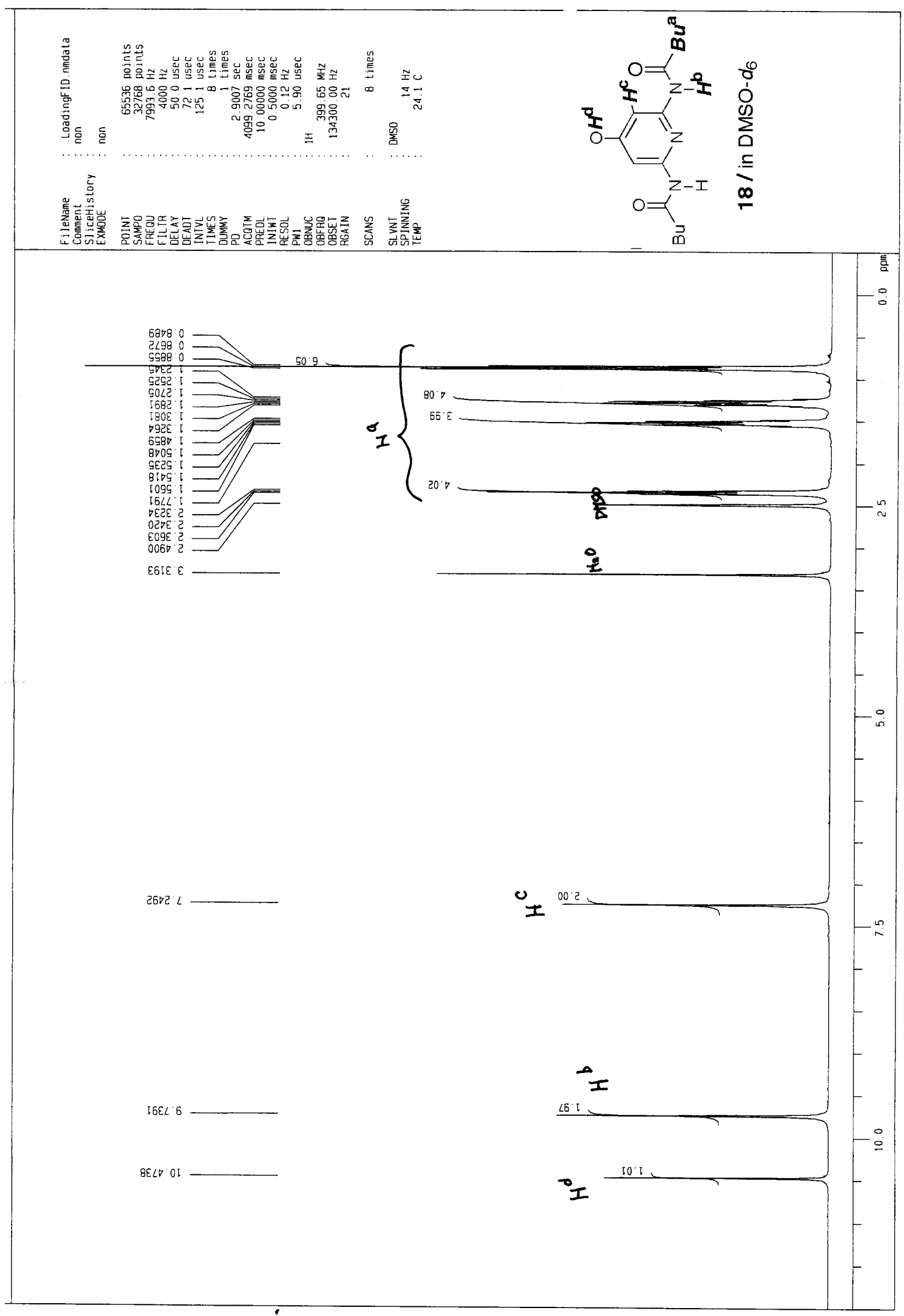




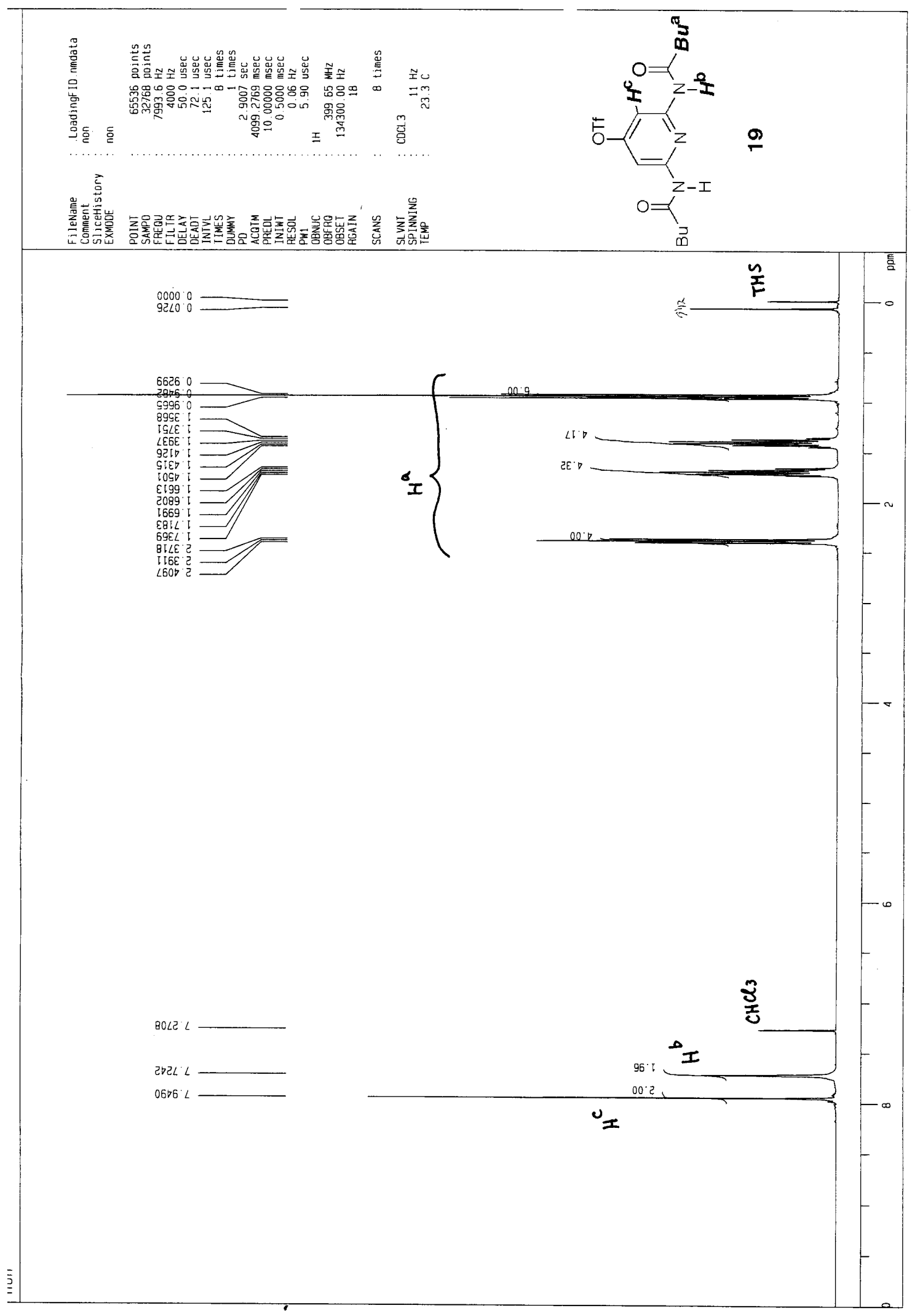




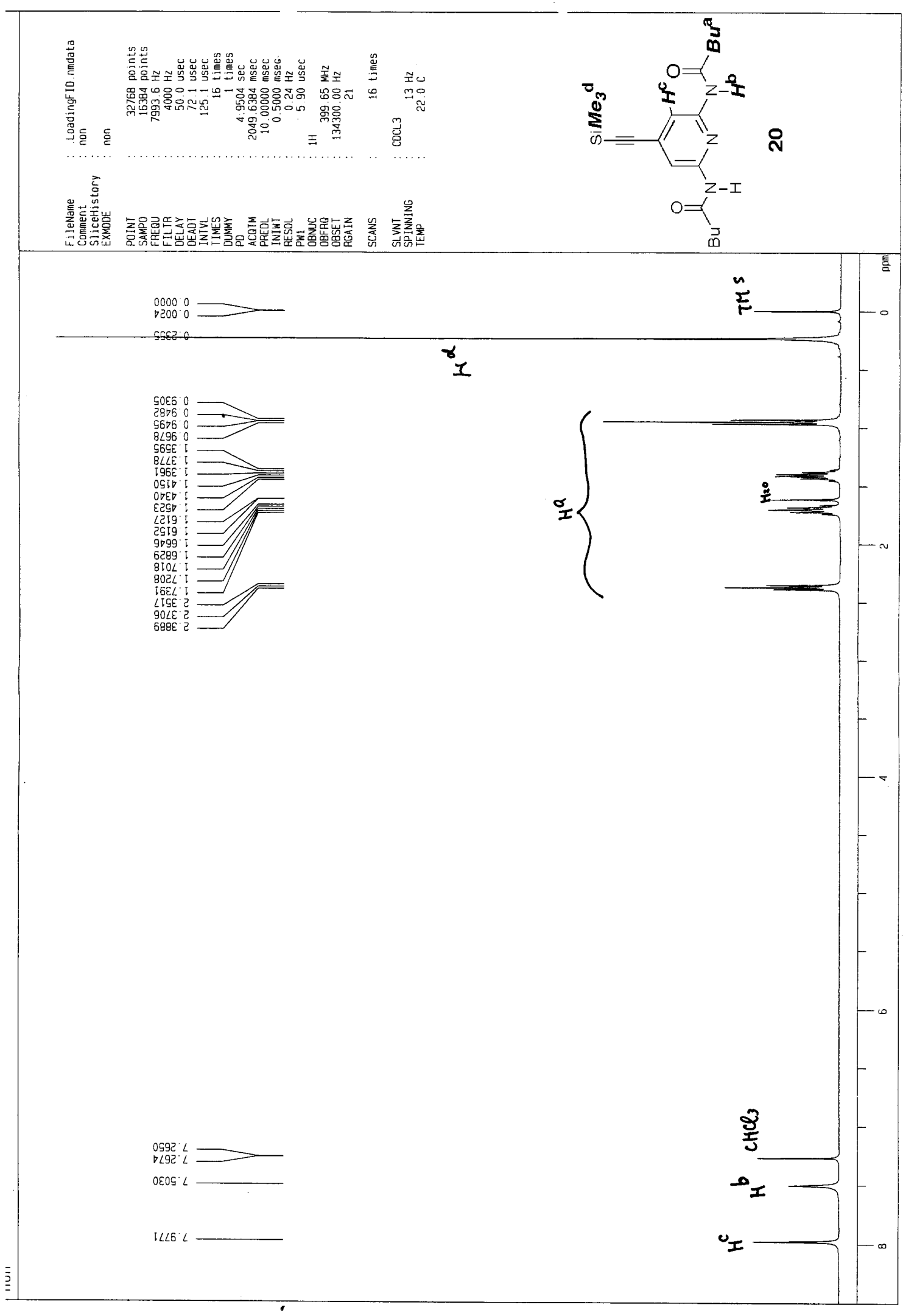




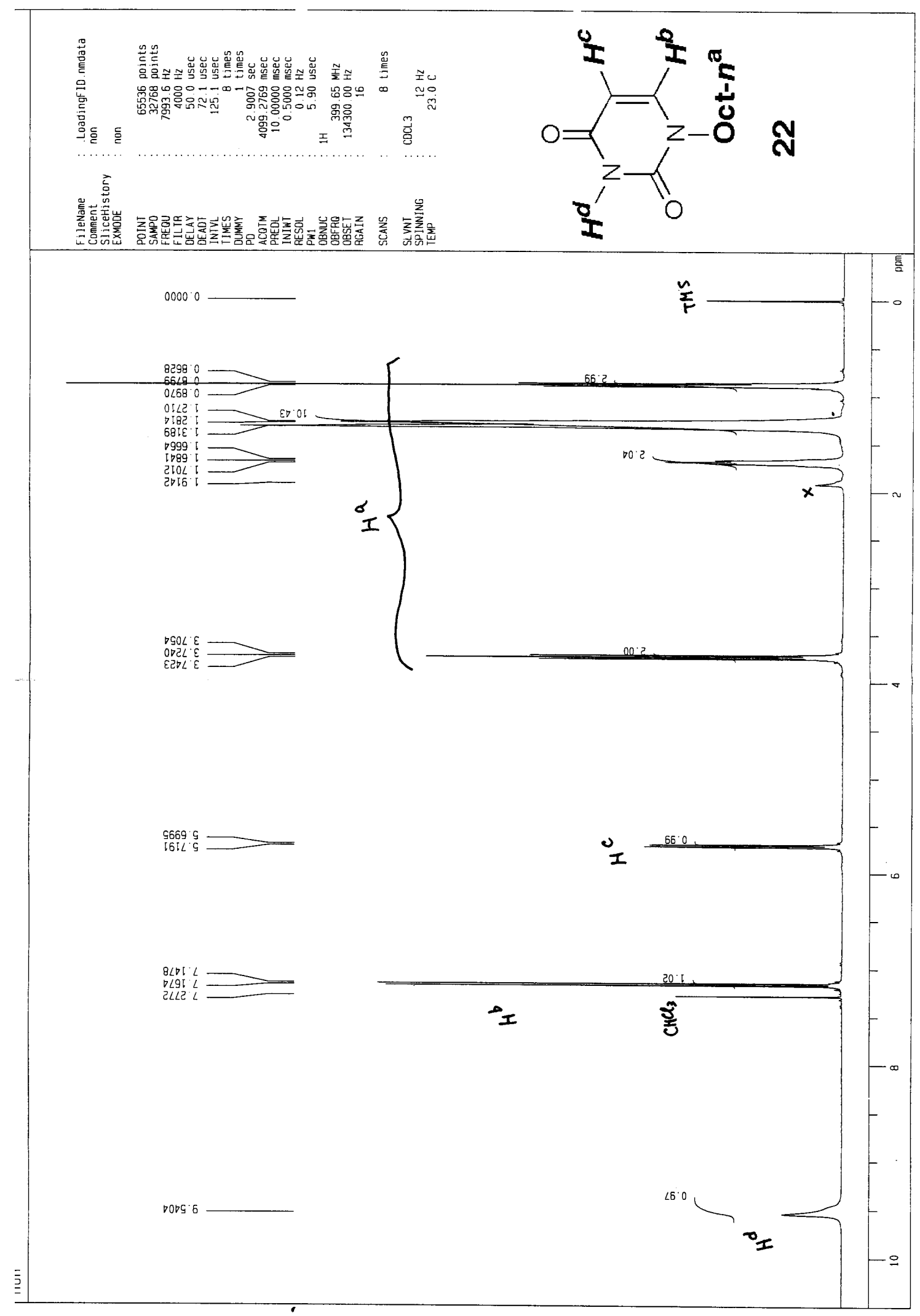




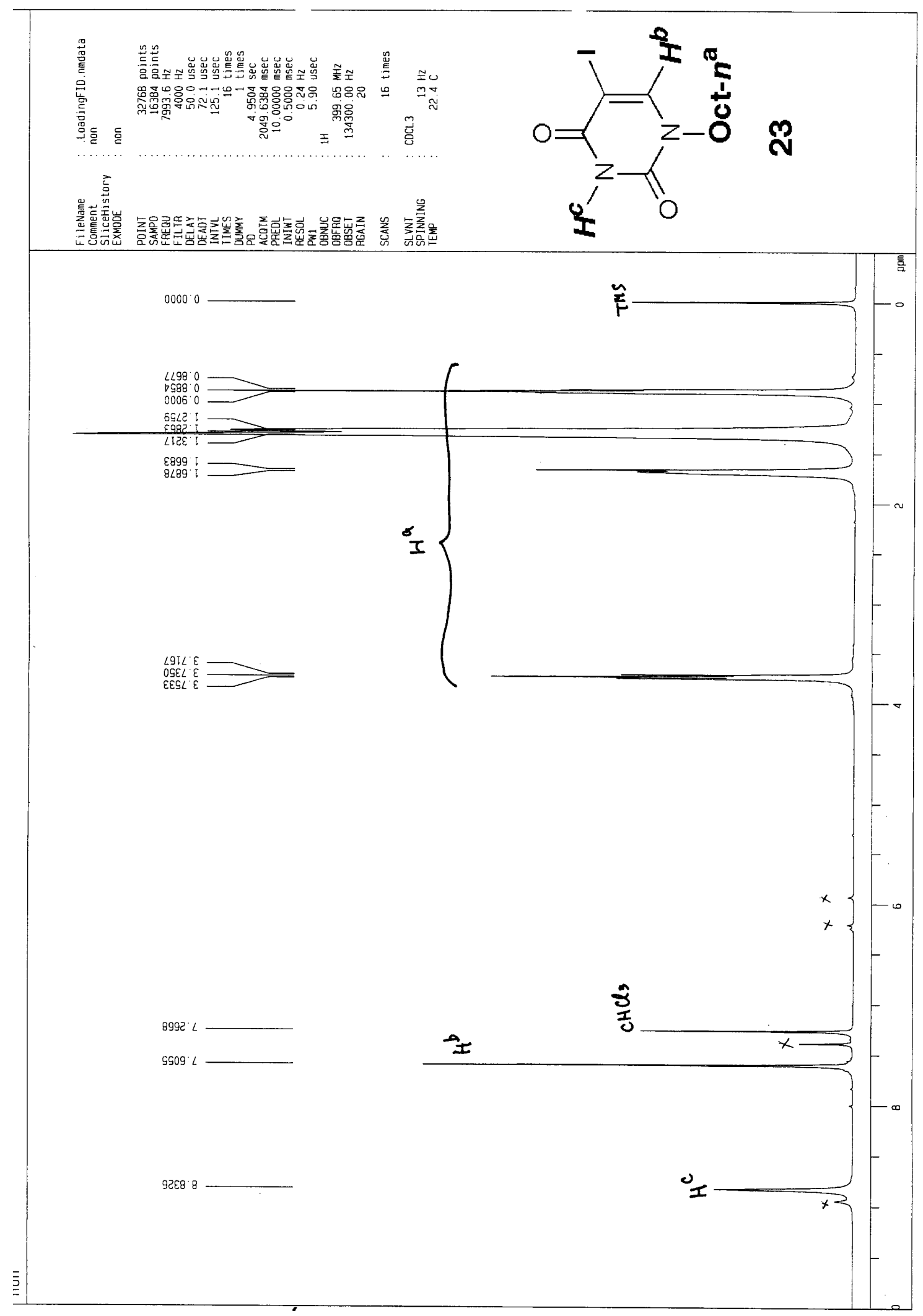




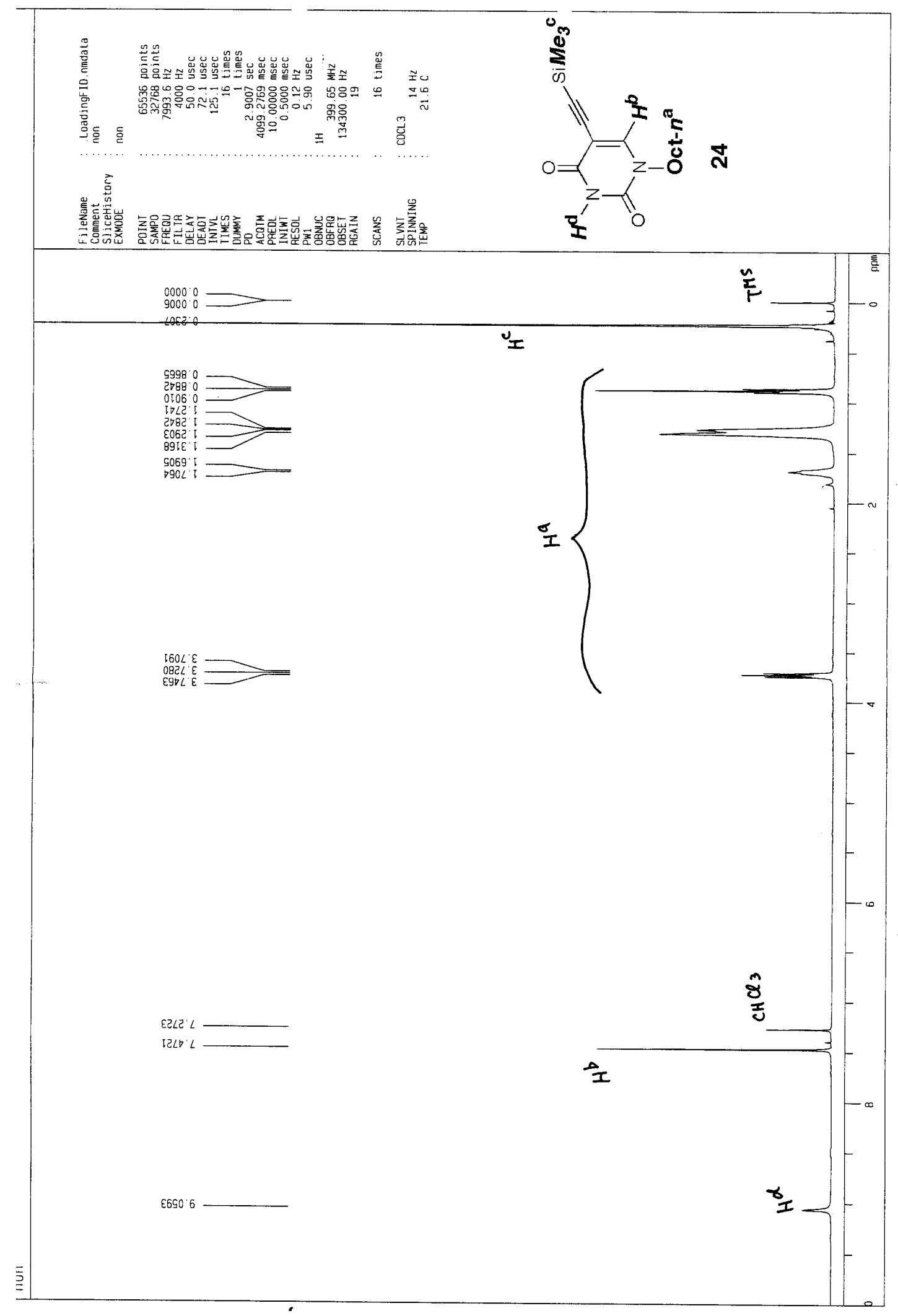




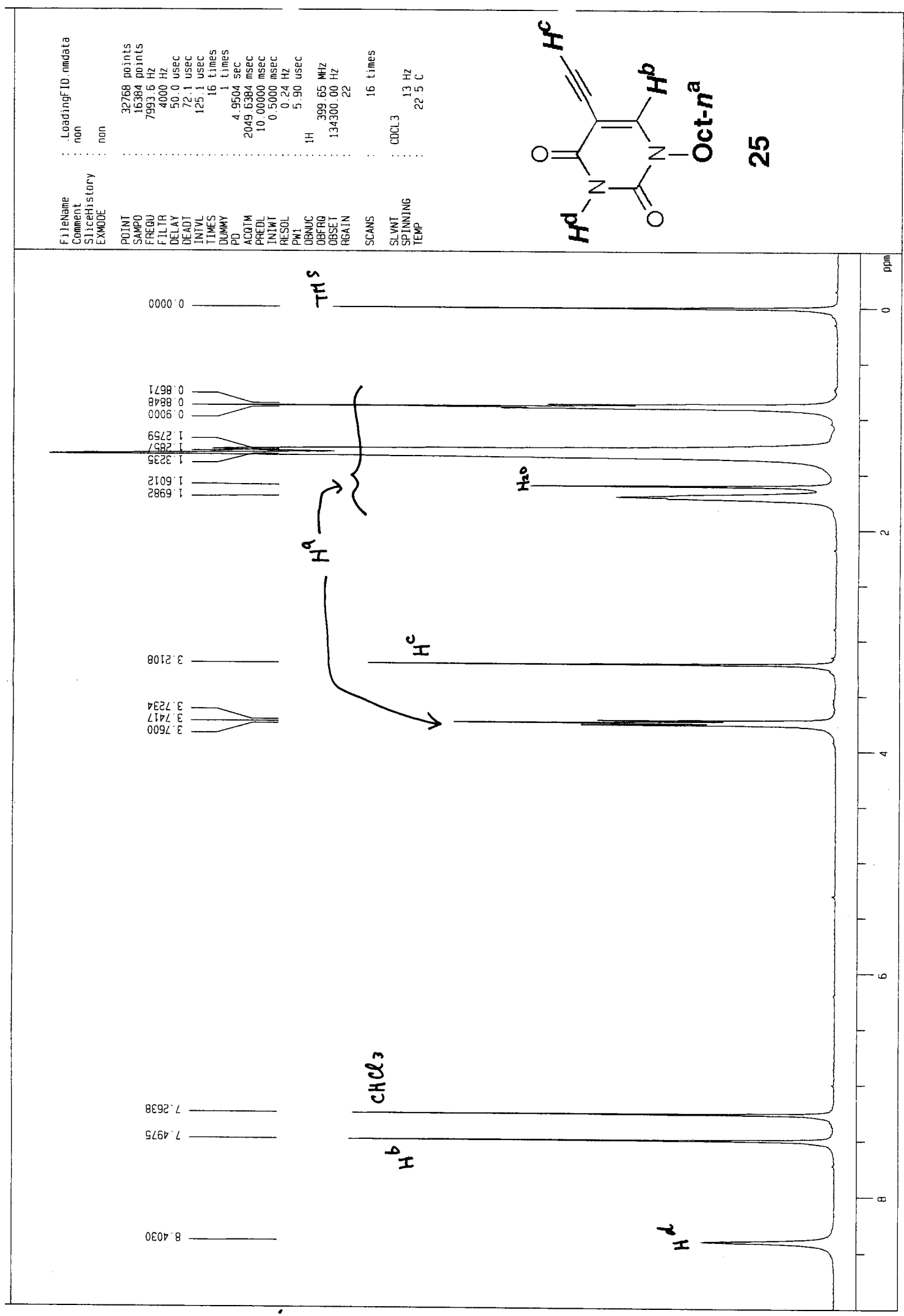




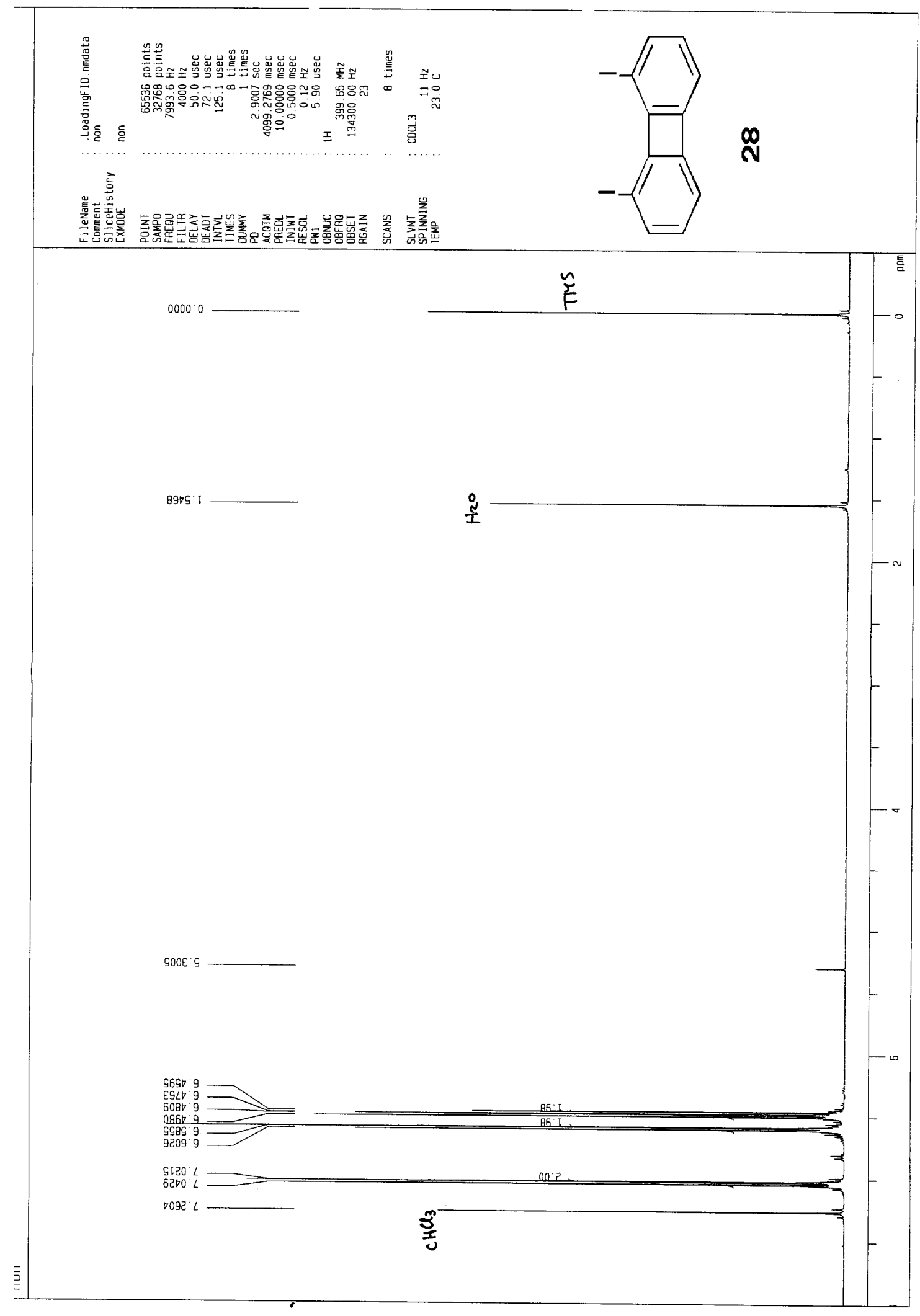

